\title{
Effects on calculated half-widths and shifts from the line coupling for asymmetric-top molecules
}

\author{
Q. Ma, ${ }^{1}$ C. Boulet, ${ }^{2}$ and R. H. Tipping ${ }^{3}$ \\ ${ }^{1}$ NASA/Goddard Institute for Space Studies and Department of Applied Physics and Applied, Mathematics, \\ Columbia University, 2880 Broadway, New York, New York 10025, USA \\ ${ }^{2}$ Institut des Sciences Moléculaires d'Orsay (ISMO), CNRS (UMR8214) et Université Paris-Sud Bât 350, \\ Campus d'Orsay F-91405, France \\ ${ }^{3}$ Department of Physics and Astronomy, University of Alabama, Tuscaloosa, Alabama 35487-0324, USA
}

(Received 8 April 2014; accepted 2 June 2014; published online 23 June 2014)

\begin{abstract}
The refinement of the Robert-Bonamy formalism by considering the line coupling for linear molecules developed in our previous studies [Q. Ma, C. Boulet, and R. H. Tipping, J. Chem. Phys. 139, 034305 (2013); 140, 104304 (2014)] have been extended to asymmetric-top molecules. For $\mathrm{H}_{2} \mathrm{O}$ immersed in $\mathrm{N}_{2}$ bath, the line coupling selection rules applicable for the pure rotational band to determine whether two specified lines are coupled or not are established. Meanwhile, because the coupling strengths are determined by relative importance of off-diagonal matrix elements versus diagonal elements of the operator $-\mathrm{iS}_{1}-\mathrm{S}_{2}$, quantitative tools are developed with which one is able to remove weakly coupled lines from consideration. By applying these tools, we have found that within reasonable tolerances, most of the $\mathrm{H}_{2} \mathrm{O}$ lines in the pure rotational band are not coupled. This reflects the fact that differences of energy levels of the $\mathrm{H}_{2} \mathrm{O}$ states are pretty large. But, there are several dozen strongly coupled lines and they can be categorized into different groups such that the line couplings occur only within the same groups. In practice, to identify those strongly coupled lines and to confine them into sub-linespaces are crucial steps in considering the line coupling. We have calculated half-widths and shifts for some groups, including the line coupling. Based on these calculations, one can conclude that for most of the $\mathrm{H}_{2} \mathrm{O}$ lines, it is unnecessary to consider the line coupling. However, for several dozens of lines, effects on the calculated half-widths from the line coupling are small, but remain noticeable and reductions of calculated half-widths due to including the line coupling could reach to 5\%. Meanwhile, effects on the calculated shifts are very significant and variations of calculated shifts could be as large as 25\%. ( 2014 AIP Publishing LLC. [http://dx.doi.org/10.1063/1.4883058]
\end{abstract}

\section{INTRODUCTION}

The modeling of the atmosphere from satellite-based, balloon-based, and Earth-based instruments requires spectroscopic parameters of atmospheric molecules, especially those of the water vapor molecule. It is obvious that the accuracy of the latter's parameters is essential for users' applications. In order to meet the accuracy requirement for the $\mathrm{H}_{2} \mathrm{O}$ molecule, the $\mathrm{H}_{2} \mathrm{O}$ spectroscopic databases have been updated many times. ${ }^{1,2}$ In these updating processes, the theoretically calculated pressure broadened half-widths and induced shifts with the Robert-Bonamy (RB) formalism ${ }^{3}$ have played an important role.

Mainly due to its practical importance, a lot of efforts have been made for years in order to improve the RB formalism. ${ }^{4-7}$ Despite all these efforts, the RB formalism still contains several basic assumptions whose applicability was not thoroughly justified. A series of recent papers ${ }^{8,9}$ have demonstrated that for simpler systems where results of the close coupling calculations ${ }^{10}$ with sophisticated potentials are available, the RB formalism significantly overestimates the half-widths.

Recently, by scrutinizing one of the main approximations (i.e., the isolated line approximation) in the RB formalism, we have found that this approximation has been applied twice by these authors in developing their formalism. ${ }^{11,12}$ First, in calculating the resolvent operator $1 /\left(\omega-L_{0}-i W\right)$, they have only considered the diagonal matrix elements of the relaxation operator W. This implies that they have neglected a required basis change within the linespace. Due to this simplification, effects from the line mixing are ignored. Second, when they applied the linked cluster theorem to remove the cutoff, they have assumed matrix elements of the operator of $\exp \left(-\mathrm{i} \mathrm{S}_{1}-\mathrm{S}_{2}\right)$ can be replaced by the exponential of the matrix elements of the operator $-i S_{1}-S_{2}$. The latter means that they have neglected other required basis change at this stage as well. (We note that in order to unambiguously represent what happens in the second simplification procedure, we have proposed in Refs. 11 and 12 to name the second basis change and its consequences as the line coupling. Readers must be careful to distinguish the difference between the line coupling and the line mixing to avoid confusion.) With this replacement, effects from the line coupling are also ignored. We have found that the criterion of the second assumption is so stringent, that for many molecular systems of interest in atmospheric applications, it cannot be satisfied. As a result, significant effects from the line coupling are completely ignored in applying this approximation. In order to remedy this 
problem, we have developed a new method to evaluate the matrix elements of the exponential of the operator and have refined the RB formalism such that the line coupling can be well taken into account. In our previous works, ${ }^{11,12}$ we have applied this method to simple linear molecular systems: Raman $\mathrm{Q}$ lines of the auto-perturbed $\mathrm{N}_{2}$ molecule and infrared $\mathrm{P}$ and $\mathrm{R}$ lines of $\mathrm{C}_{2} \mathrm{H}_{2}$ immersed in $\mathrm{N}_{2}$. In comparison with calculated half-widths derived from the RB formalism, new calculated values for these lines are significantly reduced and become closer to the measurements.

Our main goal now is to improve the accuracy of calculated half-widths and shifts for the water vapor molecule. Because $\mathrm{H}_{2} \mathrm{O}$ is an asymmetric-top molecule and it has a much more complicated spectra than linear molecules, to study the line coupling for $\mathrm{H}_{2} \mathrm{O}$ lines immersed in $\mathrm{N}_{2}$ is more difficult. By developing and testing tools, our previous studies on the linear molecular systems served as a preparation of the present investigation. In addition, in our previous works we have ignored imaginary parts of all items involved in calculations. As a result, we have not considered effects on calculated shifts from the line coupling and consequently we could not draw quantitative conclusions about these effects. In the present study, by including all the imaginary parts in calculations, we have calculated the induced shifts of $\mathrm{H}_{2} \mathrm{O}$ lines with the line coupling. It turns out that effects on the shifts are more significant than on the half-widths.

The paper is arranged as follows. In Sec. II, the main frame of the formalism with the line coupling applicable for asymmetric-top molecules such as $\mathrm{H}_{2} \mathrm{O}$ is presented. In this section, the main topics are derivations of expressions for the off-diagonal matrix elements of $S_{2 \text {,middle }}$ in terms of the twodimensional (2D) Fourier transforms and discussions of the latters' profiles. Besides, because spectra of asymmetric-top molecules are more complicated than that of linear molecules and, consequently, to determine whether two lines of interest are coupled through the line coupling or not becomes a challenge. By considering symmetry properties of the $\mathrm{D}$ matrices appearing in the expressions for $\mathrm{S}_{2 \text {,middle }}$ and analyzing the profiles of the main 2D Fourier transforms introduced, the coupling selection rules are established with which one is able to identify groups consisting of strongly coupled lines. After developing all necessary tools, in Sec. III we present numerical calculations of the half-widths and shifts for $\mathrm{H}_{2} \mathrm{O}$ lines in the pure rotational band broadened by $\mathrm{N}_{2}$. By selecting one group consisting of strongly coupled 5 lines, detailed analyses of the matrix elements for both the operator of $-\mathrm{S}_{2}$ and the operator of $\exp \left(-\mathrm{S}_{2}\right)$ are presented. For this group and other eight sample groups, calculated half-widths and shifts with the line coupling are presented together with that obtained without the line coupling. In Sec. IV, we present discussions and conclusions.

\section{THEORY}

\section{A. Diagonal matrix elements of the $S_{2}$ terms in the coordinate representation}

With the RB formalism and other line shape theories, people usually only consider the Liouville scattering $\hat{S}$ opera- tor up to its second-order expansions consisting of two terms $S_{1}$ and $S_{2}$. As expected, evaluating $S_{2}$ is more difficult than $\mathrm{S}_{1}$ and this is the main subject to investigate effects from the line coupling.

In our previous studies, we have presented general expressions for the matrix elements of $S_{2, \text { outer, },}, S_{2, \text { outer, f }}$, and $\mathrm{S}_{2, \text { middle }}$ in the coordinate representation. ${ }^{11}$ These expressions are also applicable for more complicated molecules, but will not be presented here. In addition, the diagonal property of $S_{2, \text { outer, } i}$ and $S_{2, \text { outer, }}$ within the same bands is also valid for asymmetric-top molecules. Meanwhile, the $S_{2 \text {,middle }}$ term remains as an off-diagonal matrix. For asymmetric-top molecules immersed in a linear molecule bath, the explicit expressions for the diagonal matrix elements of $S_{2, \text { outer, },}, S_{2, \text { outer, }, \text {, }}$

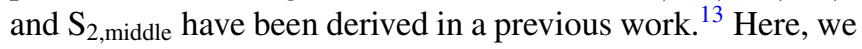
briefly outline some derivation procedures.

For a system consisting of one asymmetric-top and one linear molecule, the potential models are usually given in terms of a spherical tensor expansion as ${ }^{13}$

$$
\begin{aligned}
V(\vec{R}(t))= & \sum_{L_{1} K_{1} L_{2} L} U\left(L_{1} L_{2} L ; K_{1} ; R(t)\right) \\
& \times \sum_{m_{1} m_{2} m} C\left(L_{1} L_{2} L, m_{1} m_{2} m\right) D_{m_{1} K_{1}}^{L_{1} *}\left(\Omega_{a}\right) \\
& \times D_{m_{2} 0}^{L_{2} *}\left(\Omega_{b}\right) Y_{L m}^{*}(\omega(t)) .
\end{aligned}
$$

By introducing 2D correlation functions $G_{L_{1} K_{1} K_{1}^{\prime} L_{2}}\left(t, t^{\prime}\right)$ defined as

$$
\begin{aligned}
G_{L_{1} K_{1} K_{1}^{\prime} L_{2}}\left(t, t^{\prime}\right) & \\
= & \frac{(-1)^{K_{1}+K_{1}^{\prime}}}{4 \pi \hbar^{2}\left(2 L_{1}+1\right)^{2}\left(2 L_{2}+1\right)^{2}} \sum_{L}(-1)^{L_{1}+L_{2}+L}(2 L+1) \\
& \times U\left(L_{1} L_{2} L ; K_{1} ; R(t)\right) U\left(L_{1} L_{2} L ; K_{1}^{\prime} ; R\left(t^{\prime}\right)\right) P_{L}\left(\cos \Theta_{t, t^{\prime}}\right),
\end{aligned}
$$

and by changing their variables $t$ and $t^{\prime}$ to new variables of $\tau \equiv \mathrm{t}-\mathrm{t}^{\prime}$ and $\tau^{\prime} \equiv \frac{1}{2}\left(\mathrm{t}+\mathrm{t}^{\prime}\right)$ (i.e., $\mathrm{t}=\tau^{\prime}+\frac{1}{2} \tau$ and $\mathrm{t}^{\prime}=\tau^{\prime}$ $-\frac{1}{2} \tau$ reversely), one obtains $2 \mathrm{D}$ symmetric correlation functions $\mathbb{G}_{L_{1} K_{1} K_{1}^{\prime} L_{2}}\left(\tau, \tau^{\prime}\right)$ defined by

$$
\mathbb{G}_{L_{1} K_{1} K_{1}^{\prime} L_{2}}\left(\tau, \tau^{\prime}\right)=G_{L_{1} K_{1} K_{1}^{\prime} L_{2}}\left(\tau^{\prime}+\frac{\tau}{2}, \tau^{\prime}-\frac{\tau}{2}\right) .
$$

The great advantages of these new functions result from their symmetry properties. In contrast with the original $G_{L_{1} K_{1} K_{1}^{\prime} L_{2}}\left(t, t^{\prime}\right)$, the new $\mathbb{G}_{L_{1} K_{1} K_{1}^{\prime} L_{2}}\left(\tau, \tau^{\prime}\right)$ are even functions over the variables $\tau$ and $\tau^{\prime}$. Then, by carrying out an integration of them from $-\infty$ to $\infty$, one obtains one-dimensional correlation functions,

$$
G_{L_{1} K_{1} K_{1}^{\prime} L_{2}}(\tau)=\int_{-\infty}^{\infty} d \tau^{\prime} \mathbb{G}_{L_{1} k_{1} K_{1}^{\prime} L_{2}}\left(\tau, \tau^{\prime}\right) .
$$

Finally, in terms of $F_{L_{1} K_{1} K_{1}^{\prime} L_{2}}(\omega)$, the Fourier transforms of these one-dimensional correlation functions, one is able to obtain explicit expressions for diagonal matrix elements of

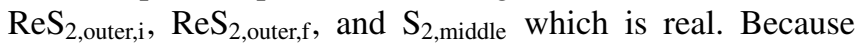
$G_{L_{1} K_{1} K_{1}^{\prime} L_{2}}(\tau)$ are even, $F_{L_{1} K_{1} K_{1}^{\prime} L_{2}}(\omega)$ are real. With respect to $\operatorname{ImS} S_{2, \text { outer, } i}$ and $\operatorname{ImS}_{2 \text {,outer, f }}$, their expressions are given in terms of the Hilbert transforms of $F_{L_{1} K_{1} K_{1}^{\prime} L_{2}}(\omega)$. Alterna- 
tively and more wisely, one can introduce the causal onedimensional correlation functions whose values have been set to zero for $\tau<0$ and find their complex Fourier transforms. ${ }^{14}$ The latter's real and imaginary parts are just the Fourier transforms $F_{L_{1} K_{1} K_{1}^{\prime} L_{2}}(\omega)$ and the subsequent Hilbert transforms, respectively. In practice, with the coordinate representation one can easily and accurately evaluate these correlation functions whose numbers are well limited. Meanwhile, based on the sampling theory, the continuous Fourier transforms are converted into their corresponding discrete Fourier transforms. With the fast Fourier transforms (FFT), ${ }^{15}$ the latter can be easily obtained. These very effective methods have been used in calculating $\mathrm{N}_{2}$ broadened half-widths and induced shifts of $\mathrm{H}_{2} \mathrm{O}$ lines based on sophistical potential models and the "exact" trajectory model ${ }^{5}$ in our previous works. $^{13,14}$

\section{B. Off-diagonal matrix elements of the $S_{2, \text { middle }}$ term}

For systems consisting of one asymmetric-top and one linear molecule, one needs to derive new expressions for

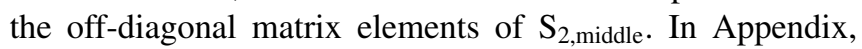
we present main steps in deriving expressions for the offdiagonal matrix elements of $\mathrm{S}_{2 \text {,middle. The derivations yield }}$ a primitive expression given by Eq. (A8) that serves as the starting point here. It turns out that the technique used to introduce the 2D correlation functions and their symmetric partners is also helpful here. However, due to interweaving variations of the variables $\mathrm{t}$ and $\mathrm{t}^{\prime}$, one has to replace $(2 L+1) P_{L}\left(\cos \Theta_{t, t^{\prime}}\right) / 4 \pi$ in Eq. (2) by their original expression of $\sum_{M} Y_{L M}^{*}\left(\theta_{\alpha}(t), \phi_{\alpha}(t)\right) Y_{L M}\left(\theta_{\beta}\left(t^{\prime}\right), \phi_{\beta}\left(t^{\prime}\right)\right)$. In other words, one prefers to rewrite the $2 \mathrm{D}$ correlation functions $G_{L_{1} K_{1} K_{1}^{\prime} L_{2}}\left(t, t^{\prime}\right)$ as

$$
\begin{aligned}
G_{L_{1} K_{1} K_{1}^{\prime} L_{2}}\left(t, t^{\prime}\right)= & \frac{(-1)^{K_{1}+K_{1}^{\prime}}}{\hbar^{2}\left(2 L_{1}+1\right)^{2}\left(2 L_{2}+1\right)^{2}} \sum_{L M}(-1)^{L_{1}+L_{2}+L} \\
& \times U\left(L_{1} L_{2} L ; K_{1} ; R(t)\right) U\left(L_{1} L_{2} L ; K_{1}^{\prime} ; R\left(t^{\prime}\right)\right) Y_{L M}^{*}\left(\theta_{\alpha}(t), \phi_{\alpha}(t)\right) Y_{L M}\left(\theta_{\beta}\left(t^{\prime}\right), \phi_{\beta}\left(t^{\prime}\right)\right) .
\end{aligned}
$$

Then, in terms of these 2D correlation functions, the expression given by (A8) can be concisely expressed as

$$
\begin{aligned}
S_{2, \text { middle }}^{i^{\prime} f^{\prime}, i f}\left(r_{c}\right)= & (-1)^{j_{i}+j_{i}^{\prime}} \sqrt{\left(2 j_{i}^{\prime}+1\right)\left(2 j_{f}^{\prime}+1\right)\left(2 j_{i}+1\right)\left(2 j_{f}+1\right)} \\
& \times \sum_{L_{1} K_{1} K_{1}^{\prime} L_{2}}(-1)^{1+J+L_{1}} W\left(j_{i}^{\prime} j_{f}^{\prime} j_{i} j_{f}, J L_{1}\right) D\left(v_{i}^{\prime} j_{i}^{\prime} \tau_{i}^{\prime} v_{i} j_{i} \tau_{i} ; L_{1} K_{1}\right) D\left(v_{f} j_{f} \tau_{f} v_{f}^{\prime} j_{f}^{\prime} \tau_{f}^{\prime} ; L_{1} K_{1}^{\prime}\right) \\
& \times \sum_{i_{2} i_{2}^{\prime}}\left(2 i_{2}+1\right)\left(2 i_{2}^{\prime}+1\right) \rho_{i_{2}} C^{2}\left(i_{2} i_{2}^{\prime} L_{2}, 000\right) \\
& \times \int_{-\infty}^{\infty} d t \int_{-\infty}^{\infty} d t^{\prime} e^{i\left(\omega_{i^{\prime}}+\omega_{i_{2}^{\prime} i_{2}}\right) t} e^{i\left(\omega_{f f^{\prime}}+\omega_{i_{i} i_{2}^{\prime}}\right) t^{\prime}} G_{L_{1} K_{1} K_{1}^{\prime} L_{2}}\left(t, t^{\prime}\right) .
\end{aligned}
$$

Furthermore, in order to exploit the advantages of the 2D symmetric correlation functions defined by Eq. (3), the integrations over $\mathrm{t}$ and $\mathrm{t}^{\prime}$ in Eq. (6) can be expressed as

$$
\begin{aligned}
& \int_{-\infty}^{\infty} \int_{-\infty}^{\infty} d t d t^{\prime} e^{i\left(\omega_{i^{\prime} i}+\omega_{i_{2}^{\prime} i_{2}}\right) t+i\left(\omega_{f f^{\prime}}+\omega_{i_{2} i_{2}}\right) t^{\prime}} G_{L_{1} K_{1} K_{1}^{\prime} L_{2}}\left(t, t^{\prime}\right) \\
& \quad=\int_{-\infty}^{\infty} \int_{-\infty}^{\infty} d \tau d \tau^{\prime} e^{i\left(\frac{\left(\omega_{\left.i^{\prime} i^{\prime}+\omega_{f^{\prime}}\right)}\right)}{2}+\omega_{i_{2}^{\prime} i_{2}}\right) \tau+i\left(\omega_{f i}-\omega_{f^{\prime} i^{\prime}}\right) \tau^{\prime}} \mathbb{G}_{L_{1} K_{1} K_{1}^{\prime} L_{2}}\left(\tau, \tau^{\prime}\right)
\end{aligned}
$$

Except for necessary modifications resulting from considering asymmetric-top molecules, this expression is very similar to that obtained in our previous study for infrared $\mathrm{P}$ and $\mathrm{R}$ lines of linear molecules because the line coupling could also occur between lines in different branches. As a result, after making the modifications, all the proce- dures, including the introduction of the 2D Fourier transforms, developed previously in evaluating the off-diagonal

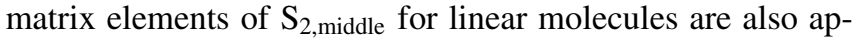
plicable for asymmetric-top molecules. Here, we only present the final expression for the off-diagonal matrix elements of $\mathrm{S}_{2 \text {,middle }}$ as 


$$
\begin{aligned}
S_{2, \text { middle }}^{i^{\prime} f^{\prime}, i f}\left(r_{c}\right)= & 2 \pi(-1)^{j_{i}+j_{i}^{\prime}} \sqrt{\left(2 j_{i}^{\prime}+1\right)\left(2 j_{f}^{\prime}+1\right)\left(2 j_{i}+1\right)\left(2 j_{f}+1\right)} \\
& \times \sum_{L_{1} K_{1} K_{1}^{\prime} L_{2}}(-1)^{1+J+L_{1}} W\left(j_{i}^{\prime} j_{f}^{\prime} j_{i} j_{f}, J L_{1}\right) D\left(v_{i}^{\prime} j_{i}^{\prime} \tau_{i}^{\prime} v_{i} j_{i} \tau_{i} ; L_{1} K_{1}\right) D\left(v_{f} j_{f} \tau_{f} v_{f}^{\prime} j_{f}^{\prime} \tau_{f}^{\prime} ; L_{1} K_{1}^{\prime}\right) \\
& \times \sum_{i_{2} i_{2}^{\prime}}\left(2 i_{2}+1\right)\left(2 i_{2}^{\prime}+1\right) \rho_{i_{2}} C^{2}\left(i_{2} i_{2}^{\prime} L_{2}, 000\right) \mathbb{F}_{L_{1} K_{1} K_{1}^{\prime} L_{2}}\left(\frac{\omega_{i^{\prime} i}+\omega_{f^{\prime} f}}{2}+\omega_{i_{2}^{\prime} i_{2}}, \omega_{f^{\prime} i^{\prime}}-\omega_{f i}\right),
\end{aligned}
$$

where $\mathbb{F}_{L_{1} K_{1} K_{1}^{\prime} L_{2}}\left(\omega, \omega^{\prime}\right)$ are the 2D Fourier transforms of $\mathbb{G}_{L_{1} K_{1} K_{1}^{\prime} L_{2}}\left(\tau, \tau^{\prime}\right)$ defined by

$$
\begin{aligned}
& \mathbb{F}_{L_{1} K_{1} K_{1}^{\prime} L_{2}}\left(\omega, \omega^{\prime}\right) \\
& \quad=\frac{1}{2 \pi} \int_{-\infty}^{\infty} \int_{-\infty}^{\infty} d \tau d \tau^{\prime} e^{i \omega \tau} e^{i \omega^{\prime} \tau^{\prime}} \mathbb{G}_{L_{1} K_{1} K_{1}^{\prime} L_{2}}\left(\tau, \tau^{\prime}\right) .
\end{aligned}
$$

The expression given by Eq. (8) is used to carry out numerical calculations for asymmetric-top molecules immersed in the linear molecule bath.

\section{Sample profiles of the 2D symmetric correlation functions and their 2D Fourier transforms}

In the present study, we consider the line coupling within the pure rotational band of the $\mathrm{H}_{2} \mathrm{O}$ molecule immersed in $\mathrm{N}_{2}$ bath. We adopt the potential model by Gamache and Laraia ${ }^{16}$ used in their calculations of the half-widths and shifts for the HITRAN database. This is a typical complicated model containing a short-range interaction modeled by the site-site model.

With the RB formalism, the site-site model has to be transformed into its spherical expansion form first. During this process, one needs to introduce two kinds of cut-offs. ${ }^{13}$ The first is a cut-off to set the upper limits for tensor ranks $\mathrm{L}_{1}$ and $\mathrm{L}_{2}$. The second is a cut-off to limit expansion terms whose inverse powers of $\mathrm{R}(\mathrm{t})$ are not beyond certain numbers. In the present study, in order to evaluate the diagonal matrix elements of $S_{2}$, we have chosen 4 as the upper limits for $\mathrm{L}_{1}$ and $\mathrm{L}_{2}$ and selected 20th order cut-off to guarantee the convergence. With the current choice of 4 , the number of the one-dimensional correlation functions is 132 . Thanks to their symmetries, there are only 39 independent ones.

With respect to evaluations of the off-diagonal matrix elements of $S_{2 \text {,middle }}$, the corresponding 2D correlation functions are required. Based on our previous works, ${ }^{11,12}$ we know that contributions to these off-diagonal matrix elements mainly come from nearly head-on collisions. This implies that among all the 2D correlation functions selected above, those having large magnitudes in regions with small closest distances are more important than others. Besides, due to symmetry restrictions, contributions from the 2D correlation functions with $\mathrm{L}_{1}=2$ are either zero or completely canceled out in calculations. Based on these considerations, we have only considered those 2D correlations associated with three main independent ones labeled by $\left(L_{1}, K_{1} K_{1}^{\prime} L_{2}\right)=(1000),(1002)$, and (3220), respectively. Among them, the 2D correlation func- tions of (1000) and (1002) are the two main contributors. It is worth mentioning that the two leading one-dimensional correlation functions with $\mathrm{L}_{1}=1$ do not make any contributions to the diagonal matrix elements of $S_{2 \text {,middle }}$.

Recall that all these 2D correlation functions are associated with specified collisional trajectories. Usually, the latter are labeled by the closest distance $r_{c}$ (or the impact parameter b). As a result, they are also functions of $r_{c}$. With the coordinate representation, it is easy to calculate these 2D symmetric correlation functions. For shortening the paper, we do not present their profiles here. After these functions are available, one can evaluate their corresponding 2D Fourier transforms with the same tools developed previously. ${ }^{12}$ Usually, when people carry out practical calculations, they prefer to use dimensionless variables in resonance functions, and we follow their common custom here. Thus, for the 2D Fourier transforms, instead of $\omega$ and $\omega^{\prime}$, we use dimensionless variables $k \equiv \omega r_{c} / \bar{v}$ and $k^{\prime} \equiv \omega^{\prime} r_{c} / \bar{v}$, where $\bar{v}$ is the mean velocity. We note that no explicit thermal average has been performed in the present study.

In order to show their profiles, we select the Fourier transforms of $\mathbb{F}_{1000}\left(k, k^{\prime}\right)$ and $\mathbb{F}_{1002}\left(k, k^{\prime}\right)$ at $\mathrm{T}=296 \mathrm{~K}$ and choose two specified trajectories at $r_{c}=3.7 \AA$ and $4.5 \AA$ representing a nearly head-on collision and a collision between nearly head-on and glancing collisions. (Based on the potential model used here, one can determine $r_{c, \text { min }}$, the minimum of the closest distance, for the "exact" trajectory model at T $=296 \mathrm{~K}$ is $3.5224 \AA$.) In Figs. 1 and 2, we present the threedimensional profile of $\mathbb{F}_{1000}\left(k, k^{\prime}\right)$ at $\mathrm{r}_{\mathrm{c}}=3.7 \AA$ and its contour plot. These two figures demonstrate that $\mathbb{F}_{1000}\left(k, k^{\prime}\right)$ has four peaks whose heights are located at $(4,0),(-4,0),(0$, $10)$, and $(0,-10)$ in the $\mathrm{k}$ and $\mathrm{k}^{\prime}$ coordinates. In addition, their slopes along the $\mathrm{k}$ axis are deeper than their slopes along the $\mathrm{k}^{\prime}$ axis. Roughly speaking, its magnitudes become negligible beyond the region of $|\mathrm{k}| \leq 12$ and $\left|\mathrm{k}^{\prime}\right| \leq 20$. For $\mathrm{r}_{\mathrm{c}}$ $=3.7$ and $\mathrm{T}=296 \mathrm{~K}$, the value converts from $\omega$ (in $\mathrm{cm}^{-1}$ ) to $\mathrm{k}$ (dimensionless) are $\mathrm{k} \approx 0.092 \times \omega$. As a result, the above region can be also described as $|\omega| \leq 130 \mathrm{~cm}^{-1}$ and $\left|\omega^{\prime}\right|$ $\leq 217 \mathrm{~cm}^{-1}$.

Similarly, we present the three-dimensional plot and the contour plot of $\mathbb{F}_{1002}\left(k, k^{\prime}\right)$ at $3.7 \AA$ in Figs. 3 and 4. As shown in the figures, it contains two peaks narrowly located at $(0.75$, $0)$ and $(-0.75,0)$ and its magnitudes are roughly limited in the region of $|\mathrm{k}| \leq 4$ and $\left|\mathrm{k}^{\prime}\right| \leq 3$ (i.e., $|\omega| \leq 43 \mathrm{~cm}^{-1}$ and $\left|\omega^{\prime}\right|$ $\left.\leq 33 \mathrm{~cm}^{-1}\right)$. In comparison with $\mathbb{F}_{1000}\left(k, k^{\prime}\right)$, it is obvious that the magnitudes of $\mathbb{F}_{1002}\left(k, k^{\prime}\right)$ are significant smaller and are confined in the narrower region. Therefore, one can conclude 


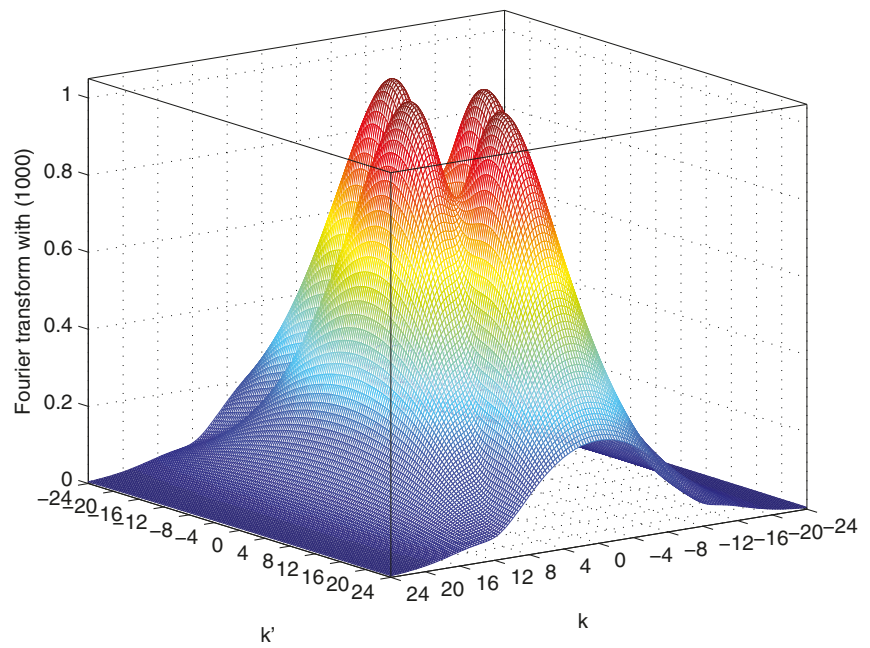

FIG. 1. The 2D Fourier transform $\mathbb{F}_{1000}\left(k, k^{\prime}\right)\left(\right.$ in $\left.\mathrm{ps}^{-2}\right)$ at $\mathrm{r}_{\mathrm{c}}=3.7 \AA$ and $\mathrm{T}$ $=296 \mathrm{~K}$ for a molecular pair of $\mathrm{H}_{2} \mathrm{O}-\mathrm{N}_{2}$. The calculation is based on the "exact" trajectory model.

that for nearly head-on collisions, $\mathbb{F}_{1000}\left(k, k^{\prime}\right)$ is more important than $\mathbb{F}_{1002}\left(k, k^{\prime}\right)$.

Furthermore, in order to show their profiles for collisions between nearly head-on and glancing collisions, we present the three-dimensional plots for $\mathbb{F}_{1000}\left(k, k^{\prime}\right)$ and $\mathbb{F}_{1002}\left(k, k^{\prime}\right)$ at $\mathrm{r}_{\mathrm{c}}=4.5 \AA$ and $\mathrm{T}=296$ in Figs. 5 and 6. In comparison with $\mathbb{F}_{1000}\left(k, k^{\prime}\right)$ at $r_{\mathrm{c}}=3.7 \AA$, the heights of $\mathbb{F}_{1000}\left(k, k^{\prime}\right)$ at $\mathrm{r}_{\mathrm{c}}=4.5$ $\AA$ are reduced by one order and its occupancy region is also reduced by five times. The latter is limited within $|\mathrm{k}| \leq 3$ and $\left|\mathrm{k}^{\prime}\right| \leq 5$ or alternatively, within $|\omega| \leq 27 \mathrm{~cm}^{-1}$ and $\left|\omega^{\prime}\right| \leq 45 \mathrm{~cm}^{-1}$ that results from the converting formula of $\mathrm{k} \approx 0.112 \times \omega$ applicable at $\mathrm{r}_{\mathrm{c}}=4.5 \AA$. This implies beyond nearly head-on collisions, $\mathbb{F}_{1000}\left(k, k^{\prime}\right)$ fades away very quickly.

In contrast, the heights of $\mathbb{F}_{1002}\left(k, k^{\prime}\right)$ are only slightly reduced and its occupancy region slightly increases (i.e., within $|\mathrm{k}| \leq 4.5$ and $\left|\mathrm{k}^{\prime}\right| \leq 4$ or within $|\omega| \leq 40 \mathrm{~cm}^{-1}$ and $\left|\omega^{\prime}\right|$ $\leq 36 \mathrm{~cm}^{-1}$ ). This implies beyond nearly head-on collisions,

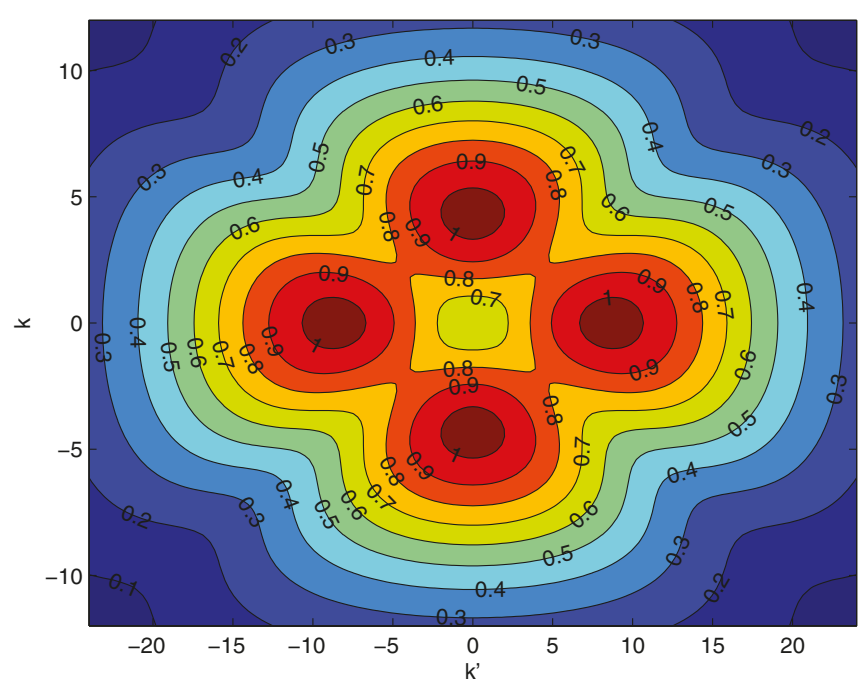

FIG. 2. The same as Fig. 1 except for the contour plot of $\mathbb{F}_{1000}\left(k, k^{\prime}\right)$ at $\mathbf{r}_{\mathrm{c}}=$ $3.7 \AA$.

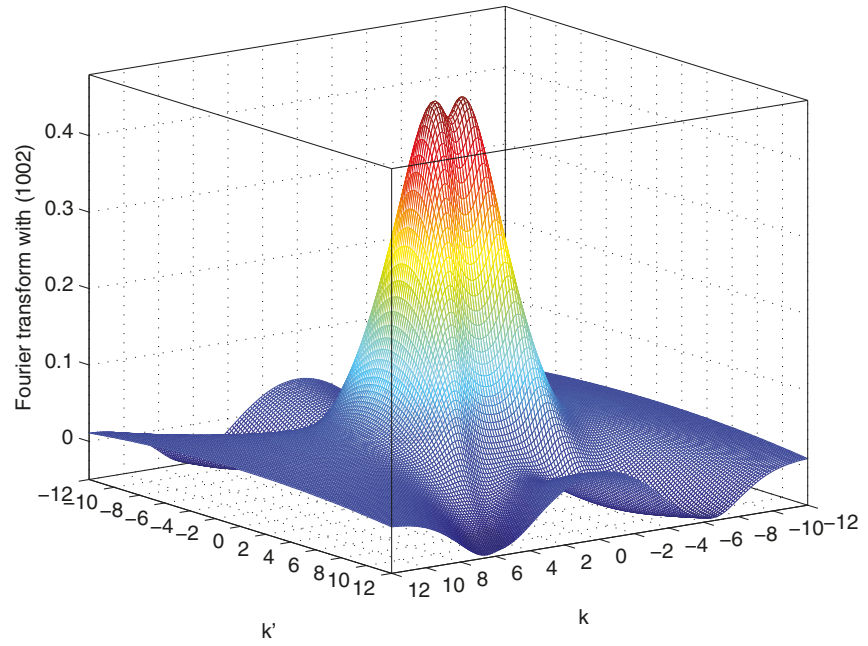

FIG. 3. The same as Fig. 1 except for the $2 \mathrm{D}$ Fourier transform $\mathbb{F}_{1002}\left(k, k^{\prime}\right)$ at $\mathrm{r}_{\mathrm{c}}=3.7 \AA$.

$\mathbb{F}_{1002}\left(k, k^{\prime}\right)$ declines slowly. In addition, in comparison with $\mathbb{F}_{1000}\left(k, k^{\prime}\right)$, both its magnitudes and occupancy region of $\mathbb{F}_{1002}\left(k, k^{\prime}\right)$ are larger. As a result, it becomes the major source responsible for the off-diagonal matrix elements.

\section{Selection rules in determining coupled $\mathrm{H}_{2} \mathrm{O}$ lines}

For linear molecules, such as $\mathrm{N}_{2}$ and $\mathrm{C}_{2} \mathrm{H}_{2}$, when one considers the line coupling, it is easy to determine their coupled lines. ${ }^{11,12}$ Usually, numbers of their intense lines are very limited and there are no couplings between the lines with even and odd $\mathrm{j}$ values. As a result, one only needs to divide the whole linespace into two sub-spaces constricted by even $\mathrm{j}$ lines and odd $\mathrm{j}$ lines, respectively. In addition, we know that for all the $\mathrm{N}_{2}$ and $\mathrm{C}_{2} \mathrm{H}_{2}$ lines, the line coupling always happens and the effects are important. For asymmetric-top molecules, such as $\mathrm{H}_{2} \mathrm{O}$, to consider the line coupling has different features. First of all, to determine groups consisting of

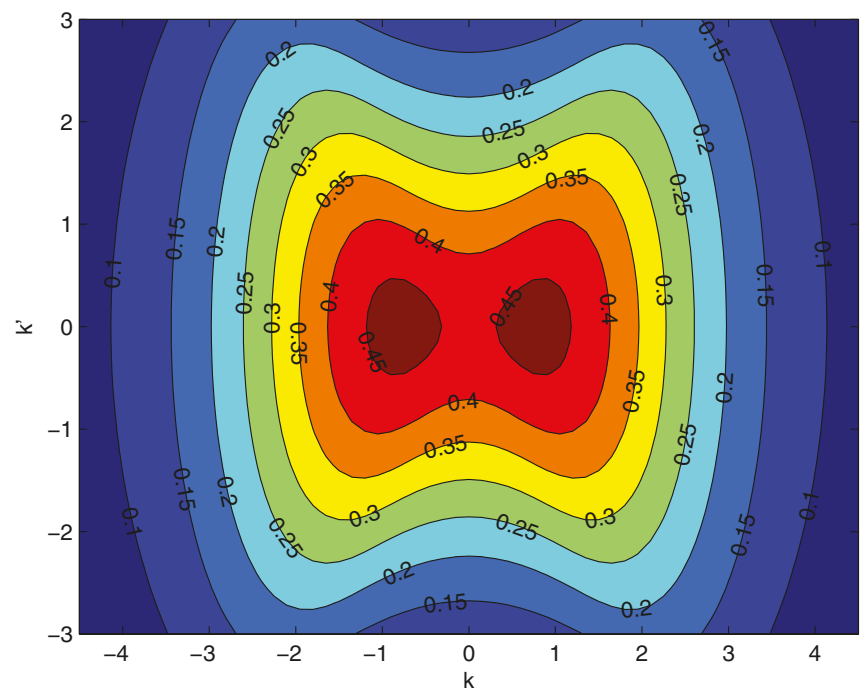

FIG. 4. The same as Fig. 2 except for the contour plot of $\mathbb{F}_{1002}\left(k, k^{\prime}\right)$ at $r_{\mathrm{c}}$ $=3.7 \AA$. 


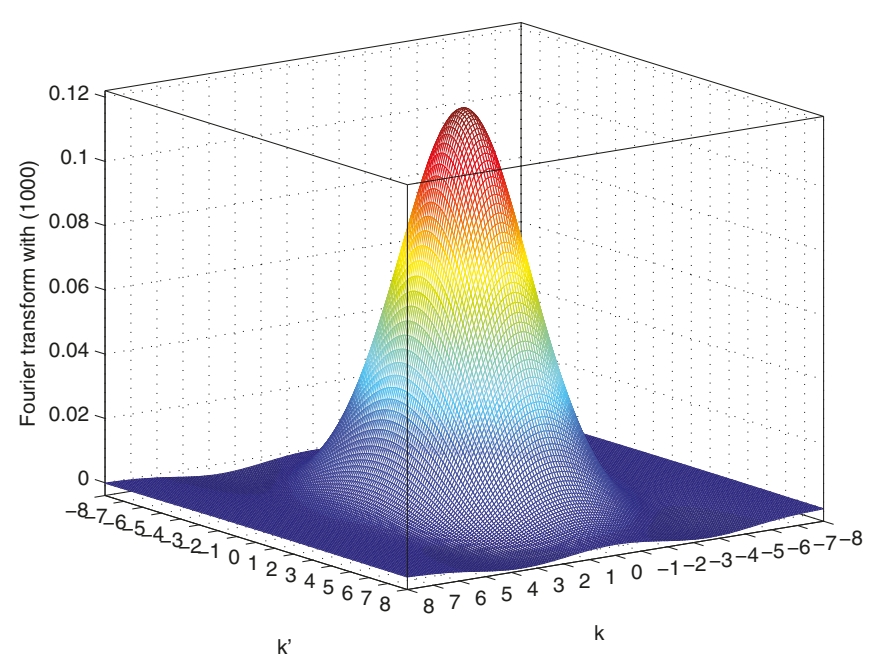

FIG. 5. The same as Fig. 1 except for $\mathbb{F}_{1000}\left(k, k^{\prime}\right)$ at $r_{\mathrm{c}}=4.5 \AA$.

coupled lines is not as simple as that for linear molecules. Second, as demonstrated later, although the line coupling could still happen between $\mathrm{H}_{2} \mathrm{O}$ lines, it would happen less often and it more likely happens less intensively.

However, based on the following considerations, one is able to find reasonable ways to identify groups of coupled lines. With Eq. (8), one can conclude that magnitudes of the off-diagonal matrix elements of $S_{2 \text {,middle }}$ between two lines of interest are mainly determined by two factors. One is the coupling strength factor defined by $\sqrt{\left(2 j_{i}^{\prime}+1\right)\left(2 j_{f}^{\prime}+1\right)\left(2 j_{i}+1\right)\left(2 j_{f}+1\right)} W\left(j_{i}^{\prime} j_{f}^{\prime} j_{i} j_{f}, J L_{1}\right)$ $D\left(v_{i}^{\prime} j_{i}^{\prime} \tau_{i}^{\prime} v_{i} j_{i} \tau_{i} ; L_{1} K_{1}\right) D\left(v_{f} j_{f} \tau_{f} v_{f}^{\prime} j_{f}^{\prime} \tau_{f}^{\prime} ; L_{1} K_{1}^{\prime}\right)$ and another is $\mathbb{F}_{L_{1} K_{1} K_{1}^{\prime} L_{2}}\left(\frac{\omega_{i^{\prime} i}+\omega_{f^{\prime} f}}{2}+\omega_{i_{2}^{\prime} i_{2}}, \omega_{f^{\prime} i^{\prime}}-\omega_{f i}\right)$. In cases that one of these factors is zero or negligible, one can ignore the line coupling between these two lines.

First of all, one can check whether the strength factor that contains a product of the two D matrices has nonzero values. For explanation convenience, we ignore the vibrational quantum numbers and rewrite the $\mathrm{D}$ matrix as $D\left(j_{k_{a}^{\prime} k_{c}^{\prime}}^{\prime} j_{k_{a} k_{c}} ; L K\right)$. There are rules to determine whether there is non-zero coupling between two states $j_{k_{a}^{\prime} k_{c}^{\prime}}^{\prime}$ and $j_{k_{a} k_{c}}$ through the $D\left(j_{k_{a}^{\prime} k_{c}^{\prime}}^{\prime} j_{k_{a} k_{c}} ; L K\right)$ matrix. For example, the rule associated with $\mathrm{L}=1$ and $\mathrm{K}=0$ is similar to the selection rule of the $\mathrm{H}_{2} \mathrm{O}$ transitions; $\Delta \mathrm{j}\left(\equiv \mathrm{j}^{\prime}-\mathrm{j}\right)=0, \pm 1, \Delta \mathrm{k}_{\mathrm{a}}\left(\equiv \mathrm{k}_{\mathrm{a}}^{\prime}-\mathrm{k}_{\mathrm{a}}\right)$ $= \pm 1, \pm 3, \cdots$, and $\Delta \mathrm{k}_{\mathrm{c}}\left(\equiv \mathrm{k}_{\mathrm{c}}^{\prime}-\mathrm{k}_{\mathrm{c}}\right)= \pm 1, \pm 3, \cdots$. In the present study, we choose the rule with $\mathrm{L}=1$ and $\mathrm{K}$ $=0$ as the selection rule because the symmetric correlation functions with (1000) and (1002) are the dominant components responsible for the off-diagonal matrix elements. In addition, because magnitudes of $D\left(j_{k_{a}^{\prime} k_{c}^{\prime}}^{\prime} j_{k_{a} k_{c}} ; 10\right)$ decrease very quickly as $\Delta \mathrm{k}_{\mathrm{a}}$ and/or $\Delta \mathrm{k}_{\mathrm{c}}$ increase, we add $\Delta \mathrm{k}_{\mathrm{a}}= \pm 1$ and $\Delta \mathrm{k}_{\mathrm{c}}= \pm 1$ as an extra restriction. Then, by enforcing these restrictions on the $\mathrm{D}$ matrices of $D\left(j_{i k_{a i}^{\prime}}^{\prime} k_{c i}^{\prime} j_{i k_{a i} k_{c i}} ; L_{1} K_{1}\right)$ and $D\left(j_{f k_{a f} k_{c f}} j_{f k_{a f}^{\prime} k_{c f}^{\prime}}^{\prime} ; L_{1} K_{1}\right)$ associated with their initial and final states of two specified lines $j_{f k_{a f} k_{c f}} \leftarrow j_{i k_{a i} k_{c a}}$ and $j_{f k_{a f}^{\prime} k_{c f}^{\prime}}^{\prime}$ $\leftarrow j_{i k_{i i}^{\prime} k_{c i}^{\prime}}^{\prime}$, one can determine whether they can be coupled or not. More specifically, both their initial and final angular moments must satisfy $\mathrm{j}_{\mathrm{i}}^{\prime}-\mathrm{j}_{\mathrm{i}}=0, \pm 1$ and $\mathrm{j}_{\mathrm{f}}^{\prime}-\mathrm{j}_{\mathrm{f}}=0, \pm 1$.

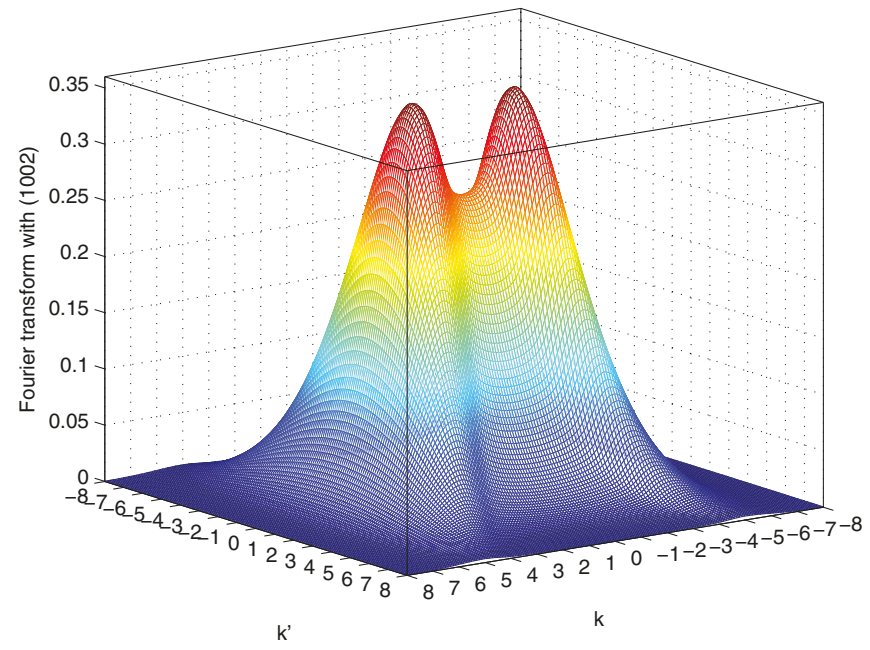

FIG. 6. The same as Fig. 1 except for $\mathbb{F}_{1002}\left(k, k^{\prime}\right)$ at $r_{c}=4.5 \AA$.

Meanwhile, their initial $\mathrm{k}_{\mathrm{a}}$ and $\mathrm{k}_{\mathrm{c}}$ quantum numbers must satisfy $\mathrm{k}_{\mathrm{ai}}^{\prime}-\mathrm{k}_{\mathrm{ai}}= \pm 1$ and $\mathrm{k}_{\mathrm{ci}}^{\prime}-\mathrm{k}_{\mathrm{ci}}= \pm 1$ and their final $\mathrm{k}_{\mathrm{a}}$ and $\mathrm{k}_{\mathrm{c}}$ quantum numbers must satisfy $\mathrm{k}_{\mathrm{af}}^{\prime}-\mathrm{k}_{\mathrm{af}}= \pm 1$ and $\mathrm{k}_{\mathrm{cf}}^{\prime}-\mathrm{k}_{\mathrm{cf}}= \pm 1$ as well. In terms of the indices $\tau_{\mathrm{i}} \equiv \mathrm{k}_{\mathrm{ai}}-\mathrm{k}_{\mathrm{ci}}$ and $\tau_{\mathrm{f}} \equiv \mathrm{k}_{\mathrm{af}}-\mathrm{k}_{\mathrm{cf}}$, the $\tau_{\mathrm{i}}$ values of their initial states and also the $\tau_{\mathrm{f}}$ values of their final states for two coupled lines must have the same evenness or oddness. Here, it is worth mentioning that due to the dipole transition selection rule, for each of the transition lines of $\mathrm{H}_{2} \mathrm{O}$, its $\tau_{\mathrm{i}}$ and $\tau_{\mathrm{f}}$ values always have the same evenness and oddness. Finally, after enforcing this selection rule, there are no more restrictions required from $W\left(j_{i}^{\prime} j_{f}^{\prime} j_{i} j_{f}, J L_{1}\right)$.

Armed with the selection rules, we have screened all pairs of $\mathrm{H}_{2} \mathrm{O}$ lines in the pure rotational band listed in HITRAN $2008^{1}$ and obtained a complete list of the coupled pairs. Most of lines have several coupled partners, but some lines are only coupled with one line. By tracing all connections through the line coupling, it turns out that the whole linespace is divided into two completely isolated sub-linespaces: one consists of lines with even $\tau_{\mathrm{i}}$ ( or $\tau_{\mathrm{f}}$ ) values and another consists of odd $\tau_{\mathrm{i}}$ (or $\tau_{\mathrm{f}}$ ) values. Within a given sub-linespaces, all lines are connected. Their connections could be established directly through the line coupling between them or not directly through a series of couplings with other intermediate lines. Meanwhile, there are no connections existing between lines that belong to different sub-linespaces. Given the fact that there are $1639 \mathrm{H}_{2} \mathrm{O}$ lines listed and sizes of the two sublinespaces are roughly the same, one has to take further steps to reduce the number of lines to be considered. One of them is to calculate the coupling strength factor first. Because the off-diagonal elements are simply proportional to its values, one can effectively rule out many weakly coupled pairs from considerations.

Then, by examining the second factor, one can develop a screening tool to easily and effectively identify strongly coupled lines. From profiles of $\mathbb{F}_{1002}\left(k, k^{\prime}\right)$ and $\mathbb{F}_{1000}\left(k, k^{\prime}\right)$ described in Sec. II C, one knows that non-negligible contributions to the off-diagonal elements of $\mathrm{S}_{2 \text {,middle }}$ from these functions come only from the relatively narrow regions of $\mathrm{k}$ and $\mathrm{k}^{\prime}$ (or alternatively, $\omega$ and $\omega^{\prime}$ ). Besides, the energy levels of 
TABLE I. Group 1 of coupled $\mathrm{H}_{2} \mathrm{O}$ lines.

\begin{tabular}{lccccc}
\hline \hline Lines & (1) $15_{87} \leftarrow 15_{78}$ & (2) $15_{78} \leftarrow 15_{69}$ & (3) $15_{69} \leftarrow 15_{510}$ & (4) $15_{510} \leftarrow 15_{411}$ & (5) $15_{411} \leftarrow 15_{312}$ \\
\hline $\mathrm{F}\left(\mathrm{cm}^{-1}\right)$ & 195.902 & 156.215 & 112.281 & 115.999 & 164.422 \\
$\mathrm{E}_{\mathrm{i}}\left(\mathrm{cm}^{-1}\right)$ & 3629.096 & 3471.881 & 3360.600 & 3244.601 & 3080.179 \\
\hline \hline
\end{tabular}

$\mathrm{H}_{2} \mathrm{O}$ states and the frequencies of $\mathrm{H}_{2} \mathrm{O}$ lines are accurately known with which one can easily calculate the averaged energy gap of $\frac{\omega_{i^{\prime} i}+\omega_{f^{\prime} f}}{2}$ (denoted later by $\left.\left(\Delta \mathrm{E}_{\mathrm{i}}+\Delta \mathrm{F}_{\mathrm{f}}\right) / 2\right)$ and the frequency gap of $\omega_{f^{\prime} i^{\prime}}-\omega_{f i}$ (denoted later by $\Delta \mathrm{F}$ ) for two coupled lines. Because the energy gaps between different $\mathrm{H}_{2} \mathrm{O}$ states are pretty large, both these two gaps have seldom chances to be small.

Careful readers may notice that the first argument of $\mathbb{F}_{L_{1} K_{1} K_{1}^{\prime} L_{2}}$ is not the averaged energy gap alone, but contains another term of $\omega_{i_{2}^{\prime} i_{2}}$. The latter represents a difference of the energy levels between two $\mathrm{N}_{2}$ states. For $\mathrm{L}_{2}=0$, it is easy to show $\omega_{i_{2}^{\prime} i_{2}}=0$. For $\mathrm{L}_{2}=2$, values of $\omega_{i_{2}^{\prime} i_{2}}$ vary from negative to positive with different weights, but $\omega_{i_{2}^{\prime} i_{2}}=0$ happens with a dominant chance. ${ }^{17}$ Therefore, one can ignore this term and simply use the averaged energy gap to represent the first argument. Thus, by introducing one cut-off to limit the averaged energy gap and another cut-off for the frequency gap, one can effectively exclude weakly coupled lines. To apply these cutoffs looks like to cut weak bonds between two coupled lines. Consequently, a lot of lines are excluded from the sub-spaces and the latter are broken into isolated groups.

Of course, there could be a convergence problem occurring in applying the cut-offs. More specifically, one has to verify calculated results would change only slightly as less stringent cut-offs are selected. We will return to this subject later. In summary, by carrying out the procedures mentioned above, one can identify groups consisting of strongly coupled lines, and at least for the pure rotational band, the sizes of these groups are very limited. Then, one can calculate the matrix elements of $-\mathrm{S}_{2}\left(\mathrm{r}_{\mathrm{c}}\right)$ within the sub-spaces, and by using tools to calculate the exponential of a matrix, one can correctly evaluate the matrix elements of $\exp \left\{-\mathrm{S}_{2}\left(\mathrm{r}_{\mathrm{c}}\right)\right\}$. Thus, the most difficult task in considering the line coupling for $\mathrm{H}_{2} \mathrm{O}$ lines becomes tractable.

\section{E. Diagonal matrix elements of the relaxation operator}

It is well known that the relaxation operator $\mathrm{W}$ contains all dynamical information of collisional processes. In the present study, we do not consider the whole matrix of the relaxation operator, rather focus attention on its diagonal matrix elements whose real and imaginary parts represent the pressure broadened half-widths and induced shifts, respectively. In terms of diagonal matrix elements of the exponential of the operator $-i S_{1}\left(r_{c}\right)-S_{2}\left(r_{c}\right)$, the expression for the diagonal el- ements of the relaxation operator $\mathrm{W}$ is given by ${ }^{11,12}$

$$
\begin{aligned}
W^{i f, i f}= & \frac{n_{b} \bar{v}}{2 \pi c} \int_{r_{c, \text { min }}}^{+\infty} 2 \pi\left(b \frac{d b}{d r_{c}}\right) \\
& \times d r_{c}\left\{1-\ll i f\left|e^{-i S_{1}\left(r_{c}\right)-S_{2}\left(r_{c}\right)}\right| i f \gg\right\},
\end{aligned}
$$

where $n_{b}$ is the number density of the bath molecules. For $\mathrm{H}_{2} \mathrm{O}$ lines in the pure rotational band, $\mathrm{S}_{1}\left(\mathrm{r}_{\mathrm{c}}\right)$ is zero. Then, with Eq. (10), one can conclude that the most important step in calculating the half-width and shift is to evaluate the diagonal matrix elements of $\left\langle\left\langle\right.\right.$ if $\left|\exp \left\{-S_{2}\left(r_{c}\right)\right\}\right|$ if $\rangle$. In fact, the essential difference between the usual method (without the line coupling) and the new method results from how to evaluate matrix elements for the exponential of matrix. For the former, by assuming the isolated line approximation, one simply replaces $\left\langle\left\langle\right.\right.$ if $\left|\exp \left\{-\mathrm{S}_{2}\left(\mathrm{r}_{\mathrm{c}}\right)\right\}\right|$ if $\rangle$ by $\exp \left\{-\left\langle\left\langle\right.\right.\right.$ if $\left|\mathrm{S}_{2}\left(\mathrm{r}_{\mathrm{c}}\right)\right|$ if $\left.\left.\rangle\right\rangle\right\}$. For the latter, by using tools to calculate the exponential of a matrix, one can correctly evaluates them without making this approximation.

\section{SAMPLE CALCULATIONS FOR $\mathrm{H}_{2} \mathrm{O}$ LINES BROADENED BY $\mathrm{N}_{2}$}

We present some tests of calculated half-widths and shifts of $\mathrm{H}_{2} \mathrm{O}$ lines immersed in $\mathrm{N}_{2}$ bath. By checking the coupling strength factor value and the two gap values for all coupled pairs in the pure rotational band, one can identify those strongly coupled pairs. The larger the strength factor is and the smaller the two gaps are, the stronger the coupling between them.

\section{A. A sample group of coupled $\mathrm{H}_{2} \mathrm{O}$ lines}

For example, we have found that for the pair of $15_{69}$ $\leftarrow 15_{510}$ and $15_{510} \leftarrow 15_{411}$, the strength factor is 1.0067 and the averaged energy gap and the frequency gap are $114.14 \mathrm{~cm}^{-1}$ and $3.72 \mathrm{~cm}^{-1}$, respectively. Although the energy gap is pretty large, it still remains in the region of efficiency of $\mathbb{F}_{1002}\left(\omega, \omega^{\prime}\right)$ with small $r_{c}$ values. By comparing with other pairs, its three coupling parameters indicate the offdiagonal elements of $S_{2, \text { middle }}$ between these two lines could be pretty large. Besides, their half-widths are in the intermediate range. This implies that the diagonal matrix elements of $\mathrm{S}_{2}$ are not too large. The latter is also important because what really matters for causing the line coupling is a comparison

TABLE II. The strength factor and the averaged energy and the frequency gaps for coupled lines.

\begin{tabular}{lcccc}
\hline \hline Coupled lines & Lines 1 and 2 & Lines 2 and 3 & Lines 3 and 4 & Lines 4 and 5 \\
\hline Strength factor & -0.8302 & -1.0243 & 1.0667 & 0.8298 \\
$\left|\Delta \mathrm{E}_{\mathrm{i}}+\Delta \mathrm{E}_{\mathrm{f}}\right| / 2\left(\mathrm{~cm}^{-1}\right)$ & 176.06 & 134.25 & 114.14 & 140.21 \\
$|\Delta \mathrm{F}|\left(\mathrm{cm}^{-1}\right)$ & 39.69 & 43.93 & 3.72 & 48.42 \\
\hline \hline
\end{tabular}


between the diagonal elements and the off-diagonal elements of the $S_{2}$ matrices. Based on these considerations, we expect that this pair could be strongly coupled.

After the core pair is selected, one needs to define a group consisting of the core pair and other coupled lines. By choosing the two cut-offs which are less stringent, other lines could be either coupled with $15_{69} \leftarrow 15_{510}$ or coupled with $15_{510}$ $\leftarrow 15_{411}$. One can choose proper cut-offs such that at least one more coupled line is added. After completing this step, the group consists of the two core lines in the first-order hierarchy and new members in the second-order hierarchy. Then, for each of the new members, one finds their coupled lines in the third-order hierarchy and adds them in the group. One continues this procedure until there are no more lines allowed by the cut-offs. It is obvious that the size of groups depends on the cut-offs. The less stringent the cut-offs are, the larger the size is. In practice, one needs to adjust the two cut-offs such that the number of lines in the group remains tractable. For the current case, we have found that by setting $\mid \Delta \mathrm{E}_{\mathrm{i}}$ $+\Delta \mathrm{E}_{\mathrm{f}} \mid / 2<180 \mathrm{~cm}^{-1}$ and $|\Delta \mathrm{F}|<100 \mathrm{~cm}^{-1}$, the group consists of 5 lines. Here, in order to include one more line (i.e., $15_{87} \leftarrow 15_{78}$ ) in the group, we have intentionally chosen the large value of $180 \mathrm{~cm}^{-1}$ as the first cut-off. After all the coupled lines are selected, the sub-linespace constructed by the selected lines is completed isolated from the remaining linespace and all following calculations are carried out within this isolated $5 \times 5$ sub-linespace. As shown later, it is unnecessary to include more lines in this group.
In Table I, we present the list of lines in this group including their frequencies and energy values of their initial states. Meanwhile, we also present the coupling parameters for all coupled pairs in Table II. With Table II, one can judge which of pairs have strong bonds and which have weak bonds. For example, the strongest bond occurs between Line 3 and Line 4 and the weakest two bonds occur between Line 1 and Line 2 and between Line 4 and Line 5 . Numerical tests will verify this claim later.

In order to show matrices of $-\mathrm{S}_{2}$ associated with nearly head-on collisions, we select the one at $r_{c}=3.7 \AA$ and present it in Matrix 1. This is a complex asymmetric $5 \times 5$ matrix whose non-zero off-diagonal elements match the couplings between lines shown in Table II. The two largest off-diagonal elements (i.e., $1.1598+\mathrm{i} 0$ and $1.3886+\mathrm{i} 0$ ) are at the third row and the fourth column $(3,4)$ and at the fourth row and the third column $(4,3)$. This is well consistent with the largest strength factor (i.e., 1.0667) and the two smallest gaps (i.e., $114.14 \mathrm{~cm}^{-1}$ and $3.72 \mathrm{~cm}^{-1}$ ) between Line 3 and Line 4 . On the other hand, the smallest off-diagonal elements are at $(1,2)$ and $(2,1)$, and at $(4,5)$ and $(5,4)$. Given the fact that the coupling parameters between Line 1 and Line 2 (i.e., -0.8302 , $176.06 \mathrm{~cm}^{-1}$, and $39.69 \mathrm{~cm}^{-1}$ ) and those between Line 4 and Line 5 (i.e., $0.8298,140.21 \mathrm{~cm}^{-1}$, and $48.42 \mathrm{~cm}^{-1}$ ) are less favorable to provide strong couplings, they are well expected. Finally, with respect to the diagonal elements, one can conclude that their imaginary parts are much smaller than their real parts.

Matrix 1. The matrix of $-S_{2}$ at $r_{c}=3.7 \AA$ for the group in Table I.

$$
\left|\begin{array}{ccccc}
-1.2084+i 0.2168 & -0.1808+i 0 & 0+i 0 & 0+i 0 & 0+i 0 \\
-0.2133+i 0 & -1.9845+i 0.1555 & -0.5381+i 0 & 0+i 0 & 0+i 0 \\
0+i 0 & -0.5829+i 0 & -3.0569-i 0.7546 & 1.1598+i 0 & 0+i 0 \\
0+i 0 & 0+i 0 & 1.3886+i 0 & -2.9071-i 0.2495 & 0.3927+i 0 \\
0+i 0 & 0+i 0 & 0+i 0 & 0.4190+i 0 & -1.6804+i 0.1884
\end{array}\right|
$$

Now, the task is to calculate the exponential of $-S_{2}$. In the present study, where both the calculated half-widths and shifts are considered, the matrices of $-\mathrm{S}_{2}$ are complex. The method adopted consists of several steps. The first step is by diagonalizing the matrix $-\mathrm{S}_{2}$, one finds its complex eigenvalues and complex right (or left) eigenvectors. From the former, one constructs a diagonal matrix $D$ and from the latter, one constructs a right eigenvector matrix $X_{R}$ whose columns represent the corresponding eigenvectors. Then, one finds the inverse matrix $X_{R}^{-1}$. After all these $D, X_{R}$, and $X_{R}^{-1}$ are available, the exponential of the matrix $-\mathrm{S}_{2}$ is given by

$$
e^{-S_{2}}=X_{R} e^{D} X_{R}^{-1}
$$

Matrix 2. The matrix of $\exp \left(-\mathrm{S}_{2}\right)$ at $\mathrm{r}_{\mathrm{c}}=3.7 \AA$ for the group in Table I.

$$
\left|\begin{array}{ccccc}
0.2962+i 0.0651 & -0.0385-i 0.0071 & 0.0071-i 0.0005 & 0.0022-i 0.0003 & 0.0002-i 0.0000 \\
-0.0454-i 0.0084 & 0.1566+i 0.0199 & -0.0553+i 0.0139 & -0.0254+i 0.0065 & -0.0041+i 0.0005 \\
0.0091-i 0.0007 & -0.0599+i 0.0150 & 0.0856-i 0.0626 & 0.0731-i 0.0382 & 0.0208-i 0.0048 \\
0.0033-i 0.0004 & -0.0330+i 0.0085 & 0.0875-i 0.0457 & 0.1064-i 0.0342 & 0.0513-i 0.0015 \\
0.0004-i 0.0000 & -0.0057+i 0.0008 & 0.0266-i 0.0062 & 0.0547-i 0.0016 & 0.1947+i 0.0355
\end{array}\right|
$$


Matrix 3. The matrix of $\operatorname{Re}\left\{-S_{2}\right\}$ at $r_{c}=3.7 \AA$ for group 1 .

$\left|\begin{array}{ccccc}0.2917+i 0.0642 & 0+i 0 & 0+i 0 & 0+i 0 & 0+i 0 \\ 0+i 0 & 0.1358+i 0.0213 & 0+i 0 & 0+i 0 & 0+i 0 \\ 0+i 0 & 0+i 0 & 0.0343-i 0.0322 & 0+i 0 & 0+i 0 \\ 0+i 0 & 0+i 0 & 0+i 0 & 0.0529-i 0.0135 & 0+i 0 \\ 0+i 0 & 0+i 0 & 0+i 0 & 0+i 0 & 0.1830+i 0.0349\end{array}\right|$

In Matrix 2, we present the calculated matrix $\exp \left(-\mathrm{S}_{2}\right)$. As a reference, in Matrix 3, we present the matrix adopted in the RB formalism without considering the line coupling. It is a diagonal matrix whose diagonal elements equal to exponentials of the diagonal elements of $-S_{2}$. By comparing Matrix 2 and Matrix 3, it is obvious that these two matrices are completely different.

Because the main subjects of the present study are calculated half-widths and shifts, one needs to focus attention on the diagonal elements of Matrix 2 and Matrix 3. By comparing them, one can conclude that the real parts of elements in Matrix 2, especially the third and fourth ones, are larger than their partners in Matrix 3. Given the fact that $\{1-\operatorname{Re}[\ll$ if $\left|\exp \left(-\mathrm{S}_{2}\right)\right|$ if $\left.\left.\left.\rangle\right\rangle\right]\right\}$ appear as a main part of the integrand in Eq. (10), one can expect that by considering the line coupling, calculated half-widths would be reduced.

In order to more clearly show how these differences would affect the calculated half-widths and shifts, we calculate two factors of $\left\{1-\operatorname{Re}\left[\left\langle\left\langle\right.\right.\right.\right.$ if $\left|\exp \left(-S_{2}\right)\right|$ if $\left.\left.\left.\rangle\right]\right]\right\}$ and $-\operatorname{Im}[《 /$ if $\left|\exp \left(-\mathrm{S}_{2}\right)\right|$ if $\left.\left.\left.\rangle\right\rangle\right]\right\}$ as functions of $\mathrm{r}_{\mathrm{c}}$ for Lines 2,3 , and 4. By carrying out the calculations with and without considering the line coupling, we obtain two sets of functions for each of the factors and present their profiles in Figs. 7 and 8, respectively. As shown in Figs. 7 and 8, effects on their profiles from the line coupling are significant for Lines 3 and 4 . More

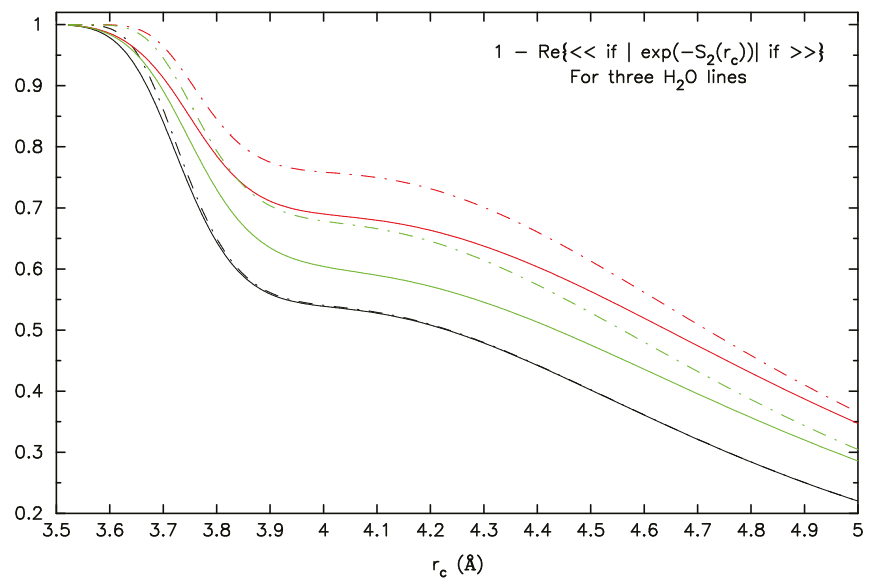

FIG. 7. Profiles of $1-\operatorname{Re}\left\{\left\langle\right.\right.$ if $\left|\exp \left(-\mathrm{S}_{2}\left(\mathrm{r}_{\mathrm{c}}\right)\right)\right|$ if $\left.\left.\rangle\right\}\right\}$ associated with the three $\mathrm{H}_{2} \mathrm{O}$ lines are plotted. They are obtained from the modified RB formalism with and without the line coupling and are plotted by three solid and dotted-dashed curves, respectively. Lines 2 , 3 , and 4 (i.e., $15_{78} \leftarrow 15_{69}$, $15_{69} \leftarrow 15_{510}$, and $\left.15_{510} \leftarrow 15_{411}\right)$ are distinguished by black, red, and green colors. explicitly, magnitudes of the first factor with the line coupling are smaller than that without the line coupling. Meanwhile, magnitudes of the second factor with the line coupling are larger than that without the line coupling. As $r_{c}$ increase, their gaps become narrower, and finally disappear. With respect to Line 2, the differences of the profiles are less noticeable. In summary, one should expect that for Line 3 and 4, it is necessity to consider the line coupling in evaluating the matrix of $\exp \left(-\mathrm{S}_{2}\right)$. In contrast, one does not need to consider the coupling for Line 2 at all.

Finally, after the diagonal elements $《$ if $\mid \exp \left(-\mathrm{S}_{2}\left(\mathrm{r}_{\mathrm{c}}\right)\right)$ $\mid$ if $\rangle\rangle$ as functions of $r_{c}$ are available, one can easily calculate the half-widths and shifts for the five lines. In Table III, we list these calculated half-widths $\gamma_{\mathrm{cp}}$ (in $\mathrm{cm}^{-1} \mathrm{~atm}^{-1}$ ) and shifts $\delta_{\mathrm{cp}}$ (in $10^{-3} \mathrm{~cm}^{-1} \mathrm{~atm}^{-1}$ ) together with $\gamma_{\text {nocp }}$ and $\delta_{\text {nocp }}$ derived from the RB formalism without the line coupling. As a reference, relative differences $\left|\Delta \gamma / \gamma_{\text {nocp }}\right|$ and $\left|\Delta \delta / \delta_{\text {nocp }}\right|$ are also presented. With Table III, one can conclude that for Line 3 and Line 4, by considering the line coupling, the calculated half-widths are reduced by $4.5 \%$ and $5.1 \%$, and meanwhile, their calculated shifts increase by $16.9 \%$ and $25.5 \%$, respectively. These numbers demonstrate that effects on the calculated shifts are significantly larger than the calculated halfwidths.

Finally, we would like to check whether the cut-offs used to define the group are suitable enough to yield converged results or not. One can easily check this by considering a sub-group only consisting of Line 3 and Line 4. Within the reduced $2 \times 2$ sub-linespace, we have repeated

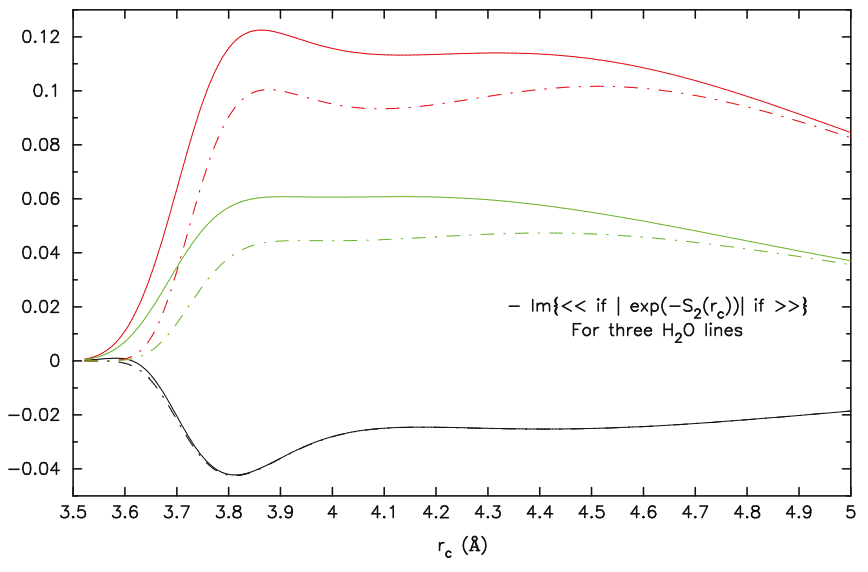

FIG. 8. The same as Fig. 7 except for $-\operatorname{Im}\left\{\left\langle\left\langle\right.\right.\right.$ if $\left|\exp \left(-\mathrm{S}_{2}\left(\mathrm{r}_{\mathrm{c}}\right)\right)\right|$ if $\left.\rangle\right\rangle$. 
TABLE III. Calculated half-widths and shifts of lines in the group listed in Table I.

\begin{tabular}{lcccccc}
\hline \hline Lines & $\gamma_{\text {nocp. }}$ & $\gamma_{\text {cp }}$ & $\left|\Delta \gamma / \gamma_{\text {nocp }}\right|(\%)$ & $\delta_{\text {nocp }} \times 10^{3}$ & $\delta_{\text {cp }} \times 10^{3}$ & $\left|\Delta \delta / \delta_{\text {nocp }}\right|(\%)$ \\
\hline $15_{87} \leftarrow 15_{78}$ & 0.0460 & 0.0458 & 0.4 & -6.025 & -6.071 & 0.8 \\
$15_{78} \leftarrow 15_{69}$ & 0.0652 & 0.0645 & 0.9 & -2.262 & -2.209 & 2.4 \\
$15_{69} \leftarrow 15_{510}$ & 0.0807 & 0.0770 & 4.5 & 7.773 & 9.084 & 16.9 \\
$15_{510} \leftarrow 15_{411}$ & 0.0750 & 0.0711 & 5.1 & 3.464 & 4.418 & 25.5 \\
$15_{411} \leftarrow 15_{312}$ & 0.0553 & 0.0550 & 0.7 & -3.453 & -3.458 & 0.1 \\
\hline \hline
\end{tabular}

previous calculations, but we do not present the calculations here except for providing some final results. For Line 3, $\gamma_{\mathrm{cp}}=0.0775 \mathrm{~cm}^{-1} \mathrm{~atm}^{-1},\left|\Delta \gamma / \gamma_{\text {nocp }}\right|=4.0 \%, \delta_{\mathrm{cp}}=8.889$ $\times 10^{-3} \mathrm{~cm}^{-1} \mathrm{~atm}^{-1}$, and $\left|\Delta \delta / \delta_{\text {nocp }}\right|=14.4 \%$. For Line 4, $\gamma_{\mathrm{cp}}=0.0715 \mathrm{~cm}^{-1} \mathrm{~atm}^{-1},\left|\Delta \gamma / \gamma_{\mathrm{nocp}}\right|=4.7 \%, \delta_{\mathrm{cp}}=4.344$ $\times 10^{-3} \mathrm{~cm}^{-1} \mathrm{~atm}^{-1}$, and $\left|\Delta \delta / \delta_{\text {nocp }}\right|=25.4 \%$. By comparing these numbers with those listed in Table IV, they are reasonably close. Therefore, one can conclude that for this group, the listed values in Table III are well converged and those obtained from the more reduced sub-linespace are also acceptable.

\section{B. Results derived from other sample groups}

We have considered dozens of groups containing strongly coupled lines. Based on our experience, strongly coupled lines, especially those with large $\mathrm{j}$ values are more likely to have small frequency gaps and relatively large average energy gaps. For the groups with low $\mathrm{j}$ values, their averaged energy gaps are smaller than that for the groups with high $\mathrm{j}$ values. This is favorable in causing the line couplings. However, the former usually have larger half-widths and larger diagonal elements of $-S_{2}$ that work against the line couplings. The net effect results from the competition of these two factors.
Meanwhile, the sizes of the groups depend on the cutoffs used and also depend on their members' $j$ values. If one applies the same cut-offs, groups consisting of lines with low $\mathrm{j}$ values have more members than that with high $\mathrm{j}$ values. This is well expected because energy level differences between different $\mathrm{H}_{2} \mathrm{O}$ states with low $\mathrm{j}$ values are smaller. However, as it has been explained above, for these groups one can apply more stringent cut-offs to limit their members. Finally, we have found that the groups to be considered could contain lines in different branches and lines could have three or more coupled partners. As a result, these groups have different sizes and have different coupling patterns. These features cause a difficulty of presentations to cover more sample groups with limited spaces.

Fortunately, as demonstrated in Sec. III A, by removing weakly coupled lines from the group, calculated results do not change too much. This convergence check enables one to simplify the presentation. In addition, because the potential model used here is not exact and there are other uncertainties existing in these semi-classical calculations, it is not our intention to provide accurate results and to cover all groups in the present study. Therefore, we prefer to use the simplest way to present results derived from these sample groups. More specifically, we have chosen eight typical groups with their $\mathrm{j}$ values covering a wide range from $\mathrm{j}=3$ to $\mathrm{j}=18$ and have decided to present results derived from their sub-groups

TABLE IV. Calculated half-widths and shifts of lines in other sample groups.

\begin{tabular}{|c|c|c|c|c|c|c|c|c|c|}
\hline Grp & Parameters & Lines & Freq. & $\gamma_{\text {nocp }}$ & $\gamma_{\mathrm{cp}}$ & $\left|\Delta \gamma / \gamma_{\text {nocp }}\right|(\%)$ & $\delta_{\text {nocp }} \times 10^{3}$ & $\delta_{\mathrm{cp}} \times 10^{3}$ & $\left|\Delta \delta / \delta_{\text {nocp }}\right|(\%)$ \\
\hline \multirow[t]{2}{*}{2} & 0.9252 & $3_{12} \leftarrow 3_{03}$ & 36.604 & 0.1075 & 0.1061 & 1.2 & 3.552 & 3.958 & 11.4 \\
\hline & $37.70,2.19$ & $3_{21} \leftarrow 3_{12}$ & 38.791 & 0.1042 & 0.1027 & 1.3 & 4.464 & 4.907 & 9.9 \\
\hline \multirow[t]{2}{*}{3} & -1.0059 & $5_{23} \leftarrow 5_{14}$ & 47.053 & 0.1020 & 0.1007 & 1.3 & 2.054 & 2.276 & 10.8 \\
\hline & $54.68,15.25$ & $5_{32} \leftarrow 5_{23}$ & 62.301 & 0.0988 & 0.0974 & 1.4 & 1.864 & 2.020 & 8.4 \\
\hline \multirow[t]{2}{*}{4} & 1.0191 & $6_{33} \leftarrow 6_{24}$ & 58.775 & 0.1002 & 0.0972 & 1.3 & 1.553 & 1.841 & 18.5 \\
\hline & $59.32,1.09$ & $6_{24} \leftarrow 6_{15}$ & 59.868 & 0.0985 & 0.0954 & 1.3 & 0.003 & 0.250 & N/A \\
\hline \multirow[t]{2}{*}{5} & 0.9536 & $7_{34} \leftarrow 7_{25}$ & 59.947 & 0.0998 & 0.0985 & 1.3 & 0.767 & 0.892 & 16.3 \\
\hline & $69.07,18.25$ & $7_{25} \leftarrow 7_{16}$ & 78.196 & 0.0922 & 0.0907 & 1.7 & -0.062 & 0.064 & N/A \\
\hline \multirow[t]{2}{*}{6} & 0.5886 & $9_{18} \leftarrow 8_{27}$ & 193.479 & 0.0598 & 0.0586 & 2.0 & -0.924 & -1.058 & 14.5 \\
\hline & $121.68,2.33$ & $9_{27} \leftarrow 8_{36}$ & 195.806 & 0.0785 & 0.0776 & 1.1 & -2.450 & -2.583 & 5.4 \\
\hline \multirow[t]{2}{*}{7} & -0.8265 & $10_{55} \leftarrow 9_{46}$ & 383.821 & 0.0747 & 0.0737 & 1.3 & 3.306 & 3.424 & 3.6 \\
\hline & $116.45,16.40$ & $10_{46} \leftarrow 9_{37}$ & 400.222 & 0.0844 & 0.0836 & 1.0 & 2.004 & 2.112 & 5.4 \\
\hline \multirow[t]{2}{*}{8} & 1.0885 & $14_{59} \leftarrow 14_{410}$ & 102.562 & 0.0821 & 0.0799 & 2.7 & 4.703 & 5.412 & 15.1 \\
\hline & $110.30,15.48$ & $14_{68} \leftarrow 14_{59}$ & 118.045 & 0.0804 & 0.0781 & 2.9 & 5.891 & 6.665 & 13.1 \\
\hline \multirow[t]{2}{*}{9} & 1.0689 & $18_{711} \leftarrow 18_{612}$ & 129.381 & 0.0710 & 0.0686 & 3.5 & 10.870 & 11.953 & 10.0 \\
\hline & $129.53,0.30$ & $18_{612} \leftarrow 18_{513}$ & 129.677 & 0.0663 & 0.0637 & 4.1 & 4.681 & 5.484 & 17.2 \\
\hline
\end{tabular}


with $2 \times 2$ sub-spaces only consisting of their core pairs in Table IV where all the quantities listed are given in the same units as those in Tables II and III. In the second column of Table IV labeled as parameters, the coupling strength factor, the averaged energy, and the frequency gaps of the groups are listed. As shown in the table, all the frequency gaps of the eight groups are below $20 \mathrm{~cm}^{-1}$, and meanwhile their averaged energy gaps, especially those with high $\mathrm{j}$ values, are much higher.

With the results listed in Table IV, one can conclude that effects on the calculated half-widths are small, but remain noticeable in the range from $1 \%$ to $4 \%$. Meanwhile, variations of the calculated shifts are significant and some of them are above $15 \%$. These conclusions are consistent with those in Sec. III A. We note that for the lines of $6_{24} \leftarrow 6_{15}$ in Group 4 and for the line of $7_{25} \leftarrow 7_{16}$ in Group 5, because their $\delta_{\text {nocp }}$ values are too small, values of $\left|\Delta \delta / \delta_{\text {nocp }}\right|$ lose their meaningfulness. In addition, we would like to note that by including more lines in these groups, the variations of $\left|\Delta \gamma / \gamma_{\text {nocp }}\right|$ and $\left|\Delta \delta / \delta_{\text {nocp }}\right|$ would increase, but the changes would not be too large.

Finally, it is worth mentioning that because the reductions and variations reported in Tables III and IV are derived from the potential model ${ }^{16}$ which is less accurate, once more accurate potentials become available and are used in calculations, these numbers could change. Therefore, it is better to treat these numbers as tentative.

\section{DISCUSSIONS AND CONCLUSIONS}

Many papers have been devoted to the line mixing for molecular lines of interest in atmospheric applications. But, few of them explicitly carry out numerical calculations for the $\mathrm{H}_{2} \mathrm{O}$ lines. The paper by $\mathrm{Lam}^{18}$ in 1977 is one of the most important. Lam considered a linespace limited to the $11 \mathrm{H}_{2} \mathrm{O}$ lines located in the microwave region. The non-diagonality of the Liouville scattering operator $\hat{S}$ within the linespace was taken into account, following an approximate procedure introduced by Dillon et al. ${ }^{19}$ Since the perturber was the $\mathrm{N}_{2}$ molecule, the potential was limited to the dipole-quadrupole interaction and the trajectories were assumed to be straight lines.

With the terminology adopted in the present study, ${ }^{11,12}$ the line mixing and the line coupling are two different concepts within the same linespaces, but they are closely related to each other. The former concerns the non-diagonality of the relaxation operator $\mathrm{W}$ appearing in the resolvent operator $1 /\left(\omega-L_{0}-i W\right)$ and the latter concerns the non-diagonality of the operator $-i S_{1}-S_{2}$ appearing in the exponential operator of $\exp \left(-\mathrm{i} \mathrm{S}_{1}-\mathrm{S}_{2}\right)$. Besides, the selection rules to determine the line mixing and the line coupling are the same. In the $\mathrm{RB}$ formalism and other formalisms where the Liouville scattering operator $\hat{S}$ is given in terms of the cumulant expansions or other exponential forms, to consider the line coupling is a prior step for considering the line mixing. In fact, after calculations of the whole matrix elements of $\exp \left(-\mathrm{i} \mathrm{S}_{1}-\mathrm{S}_{2}\right)$ are completed, one can easily derive the whole matrix elements of the relaxation operator $\mathrm{W}$. Therefore, with the method described above, we can easily check Lam's work.
In principle, as one considers the line coupling for lines located in the microwave region, one needs to take into account of both the positive-frequency lines and the negativefrequency lines. Indeed as is known from Ben-Reuven, ${ }^{20}$ positive and negative resonances are coupled by inelastic collisions. A careful analysis has shown that no efficient couplings exist between the 11 positive lines considered by Lam and their negative partners that corroborate his treatment.

In spite of his simplifying treatments of the potential and trajectory models, his four conclusions (see p. 374 of Ref. 18) are confirmed by the present work, regarding either the mixing selection rules or the weakness of the off-diagonal elements mixing some of these lines. However, by using our formalism and checking values of the line coupling parameters for those pairs considered by him, we have found that none of them are in favor of the line coupling. More specifically, among all the 11 lines, the strongest coupling happens between $4_{14} \leftarrow 3_{21}$ and $4_{23} \leftarrow 3_{30}$ and its three coupling parameters are $-0.4786,74.39 \mathrm{~cm}^{-1}$, and $2.26 \mathrm{~cm}^{-1}$. In addition, these lines have large half-width values. Indeed, we have found that reductions of the calculated half-widths for the two lines mentioned above are less than $0.5 \%$ and variations of the shifts are less than $3 \%$. As a result, the group considered by him is not a candidate to have significant effects. His conclusions are correct, but the applicability is limited. One should not apply it everywhere without exception.

Because the main targets of the present work are calculated half-widths and shifts, we draw conclusions on these subjects. First of all, with respect to the half-width, one can conclude that for most of the $\mathrm{H}_{2} \mathrm{O}$ lines in the pure rotational band, effects from the line coupling are small or negligible. This implies that for most of the $\mathrm{H}_{2} \mathrm{O}$ lines, one does not need to consider the line coupling at all. However, the above claim does not apply for a certain number of lines, or more explicitly, for several dozens of lines. For the latter, reductions of their calculated half-widths due to the line coupling are noticeable and can reach above $5 \%$ or a little bit more.

Second, with respect to the calculated shift, the situation is quite different. In general, for most of the $\mathrm{H}_{2} \mathrm{O}$ lines in the pure rotational band, effects from the line coupling are small or negligible. This means again that one does not need to consider the line coupling for them. However, for several dozens of lines or a little more, effects from the line coupling are very significant and variations of calculated shifts can reach $\pm 25 \%$ or even more. Fortunately, based on their coupling parameters and their $\mathrm{j}$ values, one can determine their identities.

In summary, our conclusions of the half-widths and shifts are different: the calculated shifts are more sensitive to the line coupling than the half-widths. It is well known that the imaginary parts of the diagonal elements of $-S_{2}$ are almost one order smaller than the real parts. Thus, in comparison with the off-diagonal elements, the former are comparable or even less and the latter are much larger. As a result, with respect to the real off-diagonal elements, relative differences of the imaginary parts and of the real parts are completely different. It turns out that the real off-diagonal elements of $-\mathrm{S}_{2}$ not only affect the real parts of the exponentials $\exp \left(-\mathrm{S}_{2}\right)$, but also the imaginary parts. Meanwhile, in both cases, the 
effects are more or less closely related to the relative differences mentioned above. Therefore, it is not surprising that the line coupling could cause larger effects on calculated shifts. Besides, values of shifts are much smaller than widths. In calculating the shift where the Hilbert transforms are involved and the latter are odd functions, there are a lot of cancellations happening and the final values result from small differences among large items involved. As a result, slight changes of values of these items could yield a large difference.

So far, we have only considered $\mathrm{H}_{2} \mathrm{O}$ lines in the pure rotational band. It is worth to extend this study to other bands, especially the $v_{2}$ band. Indeed, we expect that for this band, effects from the line coupling may be at least as significant as those for the pure rotational band. Moreover, there was a paper by Brown et al., ${ }^{21}$ where line mixing measurements for two pairs in the $v_{2}$ band were reported. One consists of two $\mathrm{P}$ branch lines (i.e., $1_{01} \leftarrow 2_{12}$ and $2_{12} \leftarrow 3_{03}$ ) and another consists of two $\mathrm{R}$ branch lines (i.e., $3_{03} \leftarrow 2_{12}$ and $2_{12} \leftarrow 1_{01}$ ). Based on the present work, one can easily verify that these authors have made good selections for their measurements because these doublets are strongly coupled. For the first pair, the three coupling parameters are $-0.9705,57.88 \mathrm{~cm}^{-1}$, and $1.24 \mathrm{~cm}^{-1}$. Meanwhile, for the second pair, the parameters are $-0.9705,55.27 \mathrm{~cm}^{-1}$, and $0.87 \mathrm{~cm}^{-1}$. (We note that because we have used the wavefunctions derived in the pure rotational band to calculate the coupling strength factors, their exact values may slightly differ from that provided above.) In general, their measured results for $\mathrm{N}_{2}$ broadened half-widths and shifts are consistent with our expectations. However, more quantitative comparisons require one to carry out numerical calculations.

Finally, our current formalism is applicable within the same band. For $\mathrm{H}_{2} \mathrm{O}$ lines belonging to different bands, the line coupling caused by intramolecular resonances (such as Coriolis, Fermi, Darling-Dennsion, and more complex resonances) could also occur. ${ }^{22}$ In this case, besides the $S_{2 \text {,middle }}$ term, both $S_{2, \text { outer, }}$ and $S_{2 \text {,outer,f }}$ can make contributions to the off-diagonal elements of $S_{2}$. More work is required to extend to these complicated cases.

\section{ACKNOWLEDGMENTS}

Two of the authors (Q. Ma and R. H. Tipping) acknowledge financial support from NSF under Grant No. 1228861. This research used resources of the National Energy Research Scientific Computing Center, which is supported by the Office of Science of the U.S. Department of Energy under Contract No. DE-AC02-05CH11231.

\section{APPENDIX: DERIVATION OF EXPRESSIONS FOR THE OFF-DIAGONAL ELEMENTS OF $\mathrm{S}_{2 \text {,middle }}$}

As shown in our previous work, by applying the cumulant expansion on the Liouville scattering $\hat{S}$ operator and introducing basic vectors in the linespace defined by

$$
\begin{aligned}
& \left.\left|i f, J M_{J}\right\rangle\right\rangle=\sum_{m_{i} m_{f}}(-1)^{j_{f}-m_{f}} C\left(j_{i} j_{f} J, m_{i}-m_{f} M_{J}\right) \mid \\
& \left.\times i m_{i} f m_{f}\right\rangle,
\end{aligned}
$$

one is able to derive general expressions for the matrix elements of $S_{2, \text { outer, }}, S_{2, \text { outer, }}$, and $S_{2 \text {,middle. Here, we show how to }}$ derive a more explicit expression for the off-diagonal matrix

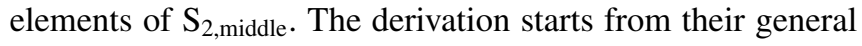
expression given by

$$
\begin{aligned}
S_{2, \text { middle }}^{i^{\prime} f^{\prime}, i f}\left(r_{c}\right)= & \frac{1}{\hbar^{2}} \int_{-\infty}^{\infty} d t \int_{-\infty}^{\infty} d t^{\prime} \ll i^{\prime} f^{\prime}, J M_{J}\left|\left\langle\mathcal{L}_{1}(t) \mathcal{L}_{1}\left(t^{\prime}\right)\right\rangle\right| i f, J M_{J} \gg_{\text {middle }} \\
= & -\frac{1}{\hbar^{2}(2 J+1)} \int_{-\infty}^{\infty} d t \int_{-\infty}^{\infty} d t^{\prime} \sum_{i_{2} m_{2}} \rho_{i_{2}} \sum_{i_{2}^{\prime} m_{2}^{\prime}} \sum_{(m)} e^{i\left(\omega_{i^{\prime} i}+\omega_{i_{2}^{\prime} i_{2}}\right) t} e^{i\left(\omega_{f f^{\prime}}+\omega_{i_{2} i_{2}^{\prime}}\right) t^{\prime}} \\
& \times \int d \Omega_{\alpha} \int d \Omega_{\beta}\left\langle i^{\prime} m_{i}^{\prime} i_{2}^{\prime} m_{2}^{\prime} \mid \alpha\right\rangle V_{\alpha}(R(t))\left\langle\alpha \mid i m_{i} i_{2} m_{2}\right\rangle \\
& \times\left\langle f m_{f} i_{2} m_{2} \mid \beta\right\rangle V_{\beta}\left(R\left(t^{\prime}\right)\right)\left\langle\beta \mid f^{\prime} m_{f}^{\prime} i_{2}^{\prime} m_{2}^{\prime}\right\rangle .
\end{aligned}
$$

For the $\mathrm{H}_{2} \mathrm{O}$ molecule, wavefunctions of its rotational states are well known. With a simple notation, they are denoted by $\left|\mathrm{j}_{\mathrm{i}} \tau_{\mathrm{i}} \mathrm{m}_{\mathrm{i}}\right\rangle$, where $\tau_{\mathrm{i}}=\mathrm{k}_{\mathrm{ai}}-\mathrm{k}_{\mathrm{ci}}$ or simply by $\left|\mathrm{i} \mathrm{m}_{\mathrm{i}}\right\rangle$. By following the convention adopted by Zare, ${ }^{23}$ Bunker and Jenson, ${ }^{24}$ the $\mathrm{H}_{2} \mathrm{O}$ wavefunctions at specified orientation labeled by $\alpha$ can be expressed as

$$
\left\langle\alpha \mid i m_{i}\right\rangle=\sqrt{\frac{2 j_{i}+1}{8 \pi^{2}}} \sum_{k} \mathcal{U}_{k \tau_{i}}^{v_{i} j_{i}}(-1)^{m_{i}-k} D_{-m_{i}-k}^{j_{i}}\left(\Omega_{a \alpha}\right) .
$$

Meanwhile, for the bath molecule of $\mathrm{N}_{2}$, the expression for its wavefunctions is given by

$$
\left\langle\alpha \mid i_{2} m_{2}\right\rangle=Y_{i_{2} m_{2}}\left(\Omega_{b \alpha}\right)
$$


With Eq. (1) and Eqs. (A3) and (A4), the integration over $\Omega_{\alpha}$ in Eq. (A2) can be expressed as

$$
\begin{aligned}
\int & d \Omega_{\alpha}\left\langle i^{\prime} m_{i}^{\prime} i_{2}^{\prime} m_{2}^{\prime} \mid \alpha\right\rangle V_{\alpha}(R(t))\left\langle\alpha \mid i m_{i} i_{2} m_{2}\right\rangle \\
= & \frac{\sqrt{\left(2 j_{i}+1\right)\left(2 j_{i^{\prime}}+1\right)}}{8 \pi^{2}} \sum_{k^{\prime} k} \mathcal{U}_{k^{\prime} \tau_{i}^{\prime}}^{v_{i}^{\prime} j_{i}^{\prime}} \mathcal{U}_{k \tau_{i}}^{v_{i} j_{i}} \sum_{L_{1} L_{2} L} \sum_{n} \sum_{\mu_{1} \mu_{2} m} U\left(L_{1} L_{2} L ; n ; R(t)\right) C\left(L_{1} L_{2} L, \mu_{1} \mu_{2} m\right) \\
& \times(-1)^{m_{i}-k}\left[\int d \Omega_{a \alpha} D_{m_{i}^{\prime} k^{\prime}}^{j_{\prime}^{\prime}}\left(\Omega_{a \alpha}\right) D_{-m_{i}-k}^{j_{i}}\left(\Omega_{a \alpha}\right) D_{\mu_{1} n}^{L_{1} *}\left(\Omega_{a \alpha}\right)\right] \\
& \times\left[\int d \Omega_{b \alpha} Y_{i_{2}^{\prime} m_{2}^{\prime}}^{*}\left(\Omega_{b \alpha}\right) Y_{i_{2} m_{2}}\left(\Omega_{b \alpha}\right) D_{\mu_{2} 0}^{L_{2} *}\left(\Omega_{b \alpha}\right)\right] Y_{L m}^{*}\left(\omega_{\alpha}(t)\right) .
\end{aligned}
$$

After analytically carrying out the integrations over $\Omega_{a \alpha}$ and $\Omega_{b \alpha}$, Eq. (A5) can be written as

$$
\begin{aligned}
\int & d \Omega_{\alpha}\left\langle i^{\prime} m_{i}^{\prime} i_{2}^{\prime} m_{2}^{\prime} \mid \alpha\right\rangle V_{\alpha}(R(t))\left\langle\alpha \mid i m_{i} i_{2} m_{2}\right\rangle \\
= & \sqrt{\left(2 j_{i}^{\prime}+1\right)\left(2 j_{i}+1\right)\left(2 i_{2}^{\prime}+1\right)\left(2 i_{2}+1\right)} \sum_{L_{1} K_{1} L_{2} L} \frac{(-1)^{K_{1}}}{\left(2 L_{1}+1\right)\left(2 L_{2}+1\right)} \\
& \times C\left(i_{2}^{\prime} i_{2} L_{2}, 000\right) U\left(L_{1} L_{2} L ; K_{1} ; R(t)\right) D\left(v_{i}^{\prime} j_{i}^{\prime} \tau_{i}^{\prime} v_{i} j_{i} \tau_{i} ; L_{1} K_{1}\right) \sum_{M_{1} M_{2} M}(-1)^{m_{i}+m_{2}} \\
& \times C\left(L_{1} L_{2} L, M_{1} M_{2} M\right) C\left(j_{i}^{\prime} j_{i} L_{1}, m_{i}^{\prime}-m_{i} M_{1}\right) C\left(i_{2}^{\prime} i_{2} L_{2}, m_{2}^{\prime}-m_{2} M_{2}\right) Y_{L M}^{*}\left(\omega_{\alpha}(t)\right) .
\end{aligned}
$$

In the above expression, the D matrix is defined by

$$
D\left(v^{\prime} j^{\prime} \tau^{\prime} v j \tau ; K L\right)=\sum_{k}(-1)^{k} \mathcal{U}_{k \tau^{\prime}}^{v^{\prime} j^{\prime}} \mathcal{U}_{k-K \tau}^{v j} C\left(j^{\prime} j L, k K-k K\right) .
$$

Similarly, one can obtain a similar expression for the integration over $\Omega_{\beta}$ in Eq. (A2). Then, with replacing the integrations over $\Omega_{\alpha}$ and $\Omega_{\beta}$ in Eq. (A2) by these two expressions and carrying out summations over all the independent magnetic quantum numbers except for $\mathrm{M}$, one is able to rewrite Eq. (A2) as

$$
\begin{aligned}
S_{2, \text { middle }}^{i^{\prime} f^{\prime}, i f}\left(r_{c}\right)= & (-1)^{j_{i}+j_{i}^{\prime}} \sqrt{\left(2 j_{i}^{\prime}+1\right)\left(2 j_{f}^{\prime}+1\right)\left(2 j_{i}+1\right)\left(2 j_{f}+1\right)} \\
& \times \sum_{L_{1} K_{1} K_{1}^{\prime} L_{2}}(-1)^{1+J+L_{1}} W\left(j_{i}^{\prime} j_{f}^{\prime} j_{i} j_{f}, J L_{1}\right) D\left(v_{i}^{\prime} j_{i}^{\prime} \tau_{i}^{\prime} v_{i} j_{i} \tau_{i} ; L_{1} K_{1}\right) D\left(v_{f} j_{f} \tau_{f} v_{f}^{\prime} j_{f}^{\prime} \tau_{f}^{\prime} ; L_{1} K_{1}^{\prime}\right) \\
& \times \sum_{i_{2} i_{2}^{\prime}}\left(2 i_{2}+1\right)\left(2 i_{2}^{\prime}+1\right) \rho_{i_{2}} C^{2}\left(i_{2} i_{2}^{\prime} L_{2}, 000\right) \frac{(-1)^{K_{1}+K_{2}}}{\hbar^{2}\left(2 L_{1}+1\right)^{2}\left(2 L_{2}+1\right)^{2}} \\
& \times \sum_{L}(-1)^{L_{1}+L_{2}+L} \sum_{M} \int_{-\infty}^{\infty} \int_{-\infty}^{\infty} d t \int_{-\infty}^{\infty} d t^{\prime} e^{i\left(\omega_{i^{\prime} i}+\omega_{i_{2}^{\prime} i_{2}}\right) t} e^{i\left(\omega_{f f^{\prime}}+\omega_{i_{2} i_{2}}\right) t^{\prime}} \\
& \times U\left(L_{1} L_{2} L ; K_{1} ; R(t)\right) U\left(L_{1} L_{2} L ; K_{1}^{\prime} ; R\left(t^{\prime}\right)\right) Y_{L M}^{*}\left(\theta_{\alpha}(t), \phi_{\alpha}(t)\right) Y_{L M}\left(\theta_{\beta}\left(t^{\prime}\right), \phi_{\beta}\left(t^{\prime}\right)\right) .
\end{aligned}
$$

${ }^{1}$ L. S. Rothman et al., JQSRT 110, 533 (2009).

${ }^{2}$ L. S. Rothman et al., JQSRT 130, 4 (2013).

${ }^{3}$ D. Robert and J. Bonamy, J. Phys. 40, 923 (1979).

${ }^{4}$ A. D. Bykov, N. N. Lavrentieva, and L. N. Sinitsa, Atmos. Oceanic Opt. 5, 587 (1992); 5, 728 (1992).

${ }^{5}$ J. Buldyreva, J. Bonamy, and D. Robert, JQSRT 62, 321 (1999).

${ }^{6}$ Q. Ma, R. H. Tipping, and C. Boulet, JQSRT 103, 588 (2007).

${ }^{7}$ Q. Ma, R. H. Tipping, and C. Boulet, J. Chem. Phys. 124, 014109 (2006).

${ }^{8}$ M. Afzelius, P.-E. Bengtsson, and J. Bonamy, J. Chem. Phys. 120, 8616 (2004).
${ }^{9}$ F. Thibault, L. Gómez, S. V. Ivanov, O. G. Buzykin, and C. Boulet, JQSRT 113, 1887 (2012); and references therein.

${ }^{10}$ S. Green, J. Chem. Phys. 62, 2271 (1975).

${ }^{11}$ Q. Ma, C. Boulet, and R. H. Tipping, J. Chem. Phys. 139, 034305 (2013).

${ }^{12}$ Q. Ma, C. Boulet, and R. H. Tipping, J. Chem. Phys. 140, 104304 (2014).

${ }^{13}$ Q. Ma, R. H. Tipping, and R. R. Gamache, Mol. Phys. 108, 2225 (2010).

${ }^{14}$ Q. Ma, R. H. Tipping, and N. N. Lavrentieva, JQRST 113, 936 (2012).

${ }^{15}$ J. W. Cooley and J. W. Tukey, Math. Comput. 19, 297-301 (1965).

${ }^{16}$ R. R. Gamache and A. L. Laraia, J. Mol. Spectrosc. 257, 116 (2009).

${ }^{17}$ Q. Ma, R. H. Tipping, and N. N. Lavrentieva, Mol. Phys. 110, 307 (2012).

${ }^{18}$ K. S. Lam, JQSRT 17, 351 (1977). 
${ }^{19}$ T. A. Dillon, E. W. Smith, J. Cooper, and M. Mizushima, Phys. Rev. A 2, 1839 (1970).

${ }^{20}$ A. Ben-Reuven, Phys. Rev. Lett. 14, 349 (1965).

${ }^{21}$ L. R. Brown, D. C. Benner, V. Malathy Devi, M. A. H. Smith, and R. A. Toth, J. Mol. Struct. 742, 111 (2005).
${ }^{22}$ A. D. Bykov, N. N. Lavrentieva, L. N. Sinitsa, and A. M. Solodov, Atmos. Oceanic Opt. 14, 774 (2001).

${ }^{23}$ R. N. Zare, Angular Momentum (Wiley, New York, 1988).

${ }^{24} \mathrm{P}$. R. Bunker and P. Jenson, Molecular Symmetry and Spectroscopy (NRC Research Press, 1998). 